\title{
Different theta frameworks coexist in the hippocampus and are coordinated during memory-guided exploration and novelty detection.
}

Víctor J. Lopez-Madrona ${ }^{1}$, Elena Pérez-Montoyo ${ }^{1}$, Efrén Álvarez-Salvado ${ }^{1}$, David Moratal ${ }^{2}$, Oscar Herreras $^{3}$, Ernesto Pereda ${ }^{4,5}$, Claudio R. Mirasso ${ }^{6}$, Santiago Canals ${ }^{1}$

1 Instituto de Neurociencias, Consejo Superior de Investigaciones Científicas, Universidad Miguel Hernández, San Juan de Alicante 03550, Spain

2 Centro de Biomateriales e Ingeniería Tisular, Universitat Politècnica de València,

$$
\text { Valencia 46022, Spain }
$$

3 Instituto Cajal, Consejo Superior de Investigaciones Científicas, Madrid 28002, Spain

4 Departamento de Ingeniería Industrial \& IUNE, Escuela Superior de Ingeniería y

Tecnología, Universidad de La Laguna, La Laguna, Tenerife 38205, Spain

5 Laboratory of Cognitive and Computational Neuroscience, Center for Biomedical

Technology, Madrid, Spain

6 Instituto de Física Interdisciplinar y Sistemas Complejos, IFISC (CSIC-UIB), Campus

Universitat de les Illes Balears, Palma de Mallorca 07122, Spain

Corresponding Author: Santiago Canals (scanals@umh.es) 


\section{Summary}

Hippocampal firing is organized in theta sequences controlled by internal memoryrelated processing and by external sensory cues. How these computations are segregated or integrated, depending on the cognitive needs, is not fully understood. Although theta activity in the hippocampus is most commonly studied as a unique coherent oscillation, it is the result of a complex interaction between different rhythm generators. Here we investigated the coordination between theta generators as a possible mechanism to couple or decouple internally and externally driven computations. We separated and quantified three different theta current generators from the hippocampus of freely behaving rats, one originating in CA3 with current sinks in CA1 str. radiatum and two with current sinks in CA1 str. lacunosummoleculare and dentate molecular layer, mainly driven by entorhinal cortex (EC) layers 3 and 2 , respectively. These theta generators followed non fully coherent dynamics and presented epochs of higher and lower phase coupling, suggesting a flexible interaction between them. Selective optogenetic inhibition in CA3 depressed the str. radiatum generator without affecting the EC-driven theta oscillations, indicating that theta rhythm generators can be modulated independently. In addition, band-specific gamma interactions with theta oscillations selectively occurred with the corresponding pathway-specific theta current generator, supporting the existence of different theta-gamma coding frameworks to organize neuronal firing in the hippocampus. Importantly, we found that epochs of highly synchronized theta rhythmicity across generators preferentially occurred during memory-guided exploration and mismatch novelty detection in familiar environments, two conditions in which internally generated memory representations need to be coordinated with the incoming sensory information about external cues. We propose a mechanism for segregating and integrating hippocampal computations based on the coexistence of different theta-gamma frameworks that flexibly couple or decouple accommodating the cognitive needs.

Keywords: hippocampus, theta, gamma, cross-frequency coupling, novelty, learning, synchronization, information transmission, binding 


\section{Introduction}

The hippocampal formation flexibly combines computations subserving spatial navigation, driven by external environmental cue stimuli (McNaughton et al., 1983; O'Keefe and Nadel, 1978), but also memory processing, dependent on internally generated firing sequences (Pastalkova et al., 2008; Wang et al., 2015). The characteristic oscillatory activity patterns in brain networks have been proposed as a mechanism to organize different computations and, depending on the cognitive needs, integrate or segregated them in oscillatory cycles (Buzsáki and Draguhn, 2004; Engel et al., 2001; Lisman and Jensen, 2013). In the hippocampus, theta and gamma oscillations are the most prominent rhythms recorded in freely moving animals (Buzsáki, 2002; Colgin, 2016, 2013; Vanderwolf, 1969). Theoretical and experimental work in the hippocampus have associated the processing of environmental cues and the encoding of memories with the input from the entorhinal cortex (EC) arriving at CA1 at a particular phase of the theta cycle, and the retrieval of memories with the CA3 output to CA1 in a different phase of the cycle (Douchamps et al., 2013; Hasselmo et al., 2002; Siegle and Wilson, 2014). Information transmission between these regions is proposed to occur in gamma oscillations of different frequencies organized in the phase of the slower CA1 theta rhythm (Colgin et al., 2009; Lisman and Idiart, 1995; Lisman and Jensen, 2013). These thetagamma associations, known as cross-frequency coupling (CFC), are modulated during exploration and memory-guided behaviours (Canolty et al., 2006; Colgin, 2015a; FernándezRuiz et al., 2017; Lasztóczi and Klausberger, 2016, 2014; Schomburg et al., 2014; Tort et al., 2008). However, theta oscillations originating in different anatomical layers of the hippocampus are known to coexist (Alonso and García-Austt, 1987; Bland and Whishaw, 1976; Buzsáki, 2002; Charpak et al., 1995; Green and Rawlins, 1979; H. Vanderwolf et al., 1973; Kramis et al., 1975; Montgomery et al., 2009; Vanderwolf, 1969; Winson, 1974) and, therefore, theta-gamma interactions need to be interpreted in the context of multiple rhythm generators.

In addition to the classical medial septum/diagonal band of Broca input imposing a global rhythmicity to the hippocampus and EC, important rhythm generators are located in EC layers II (EC2) and III (EC3), whose activity reach the dentate gyrus (DG) and hippocampus proper though the perforant and temporoammonic pathways respectively, and from CA3 activity reaching CA1 stratum radiatum through the Schafer collaterals (Buzsáki, 2002). Importantly, although theta oscillations in the hippocampus are most commonly studied as a unique coherent oscillation across hippocampal layers, exhibiting a characteristic 
amplitude/phase vs. depth variation (Buzsáki, 2002), the frequency and phase of the CA3 theta rhythm generator was shown to change relatively independently from the EC theta inputs (Kocsis et al., 1999; Montgomery et al., 2009). How these multiple theta rhythm generators and pathway-specific gamma oscillations interact in the hippocampus is not well understood. One appealing possibility is that different theta oscillations may represent different thetagamma coding frameworks, thus providing the substrate to segregate, but also to integrate different computations based on a communication-through-coherence like mechanism (Fries, 2005). A decrease in the coherence between the theta oscillations would decouple the processing streams, segregating the underlying cognitive processes (i.e. retrieval from encoding). However, an increase in the coherence would rather couple them, facilitating the integration in CA1 neurons of both information streams (i.e. when stored and ongoing contextual information need to be compared).

Here we investigated the function of pathway-specific synchronization of oscillatory activity in the hippocampus of rats freely exploring known and novel environments and resolving a T-maze. Using high density electrophysiological recordings aided by source separation techniques we characterized the dynamic properties of three different theta and three different gamma dipoles in the hippocampus with origins in the CA3 Schaffer collateral layer, the EC3 projection to the stratum lacunosum-moleculare and EC2 projection to the midmolecular layer of the DG, respectively, and found strong support for the existence of different theta-gamma frameworks. We then characterized theta-gamma interactions between the different pathways and established an association with the synchronization state in the hippocampal network. Finally, we investigated the functional role of these theta-gamma frameworks for contextual learning requiring the update of an existent memory with the changes found in the environment.

\section{Results}

\section{Pathway-specific theta and gamma oscillations}

We performed electrophysiological recordings using linear array electrodes across the dorsal hippocampus in five rats (see Supplementary Methods, Figure 1). Recordings were carried out while the animal freely explored a familiar open field (Figures 1-6), a novel open field (Figure 6) or a T-maze (Figure 7). Using spatial discrimination techniques to separate LFP sources contributed by different synaptic pathways, based on independent component 
analysis (ICA, Figure S1) (Benito et al., 2014; Fernández-Ruiz and Herreras, 2013; Herreras, 2016; Makarov et al., 2010; Makarova et al., 2011), we dissected 3 robust components in all subjects (Figure 1; Supplementary Methods). The maximum voltages (Figure 1b) and dipoles in the current source density (CSD) depth profiles (Figure 1c) of the three components matched the stratified distribution of known terminal fields in the hippocampus, and the currents resulting from stimulation of the corresponding pathways, as previously shown (Benito et al., 2014; Fernández-Ruiz and Herreras, 2013; Lasztóczi and Klausberger, 2014; Schomburg et al., 2014). The first component was located in the stratum radiatum, where the CA3 Schaffer collateral/commissural pathway targets the CA1 region (labelled as Schaffer component or Sch-IC). The second matched the EC3 projection in the stratum lacunosum-moleculare (lmIC), and the third one the perforant pathway from EC2 to the mid-molecular layer of the DG (PP-IC). These three components, referred to as pathway-specific LFPs or IC-LFPs, represent the synaptic contributions with distinct anatomical origins recorded in the LFP (Herreras, 2016).

The power spectra of these signals exhibited a clear peak at theta frequency $(6-10 \mathrm{~Hz})$ and broadband gamma activity (Figure 1e). CA3 and EC3 neurons have been shown to fire phase locked to discrete gamma band oscillations in the downstream Sch-IC and Im-IC, respectively (Fernández-Ruiz et al., 2012, p.; Schomburg et al., 2014), with gamma oscillations segregated in the phases of the theta wave recorded in CA1 (Colgin et al., 2009; Lasztóczi and Klausberger, 2014; Schomburg et al., 2014). In good agreement, pathway-specific gamma activities were distributed in the theta cycle, with $1 \mathrm{~m}-\mathrm{IC}$ close to the theta peak ( $\pi$ radians) and followed by Sch-IC (Figure 1f), showing consistency with the firing properties of principal neurons in their respective upstream afferent layers. Similarly, entorhinal principal cells in EC2 and EC3 were shown to fire in anti-phase, relative to the theta oscillation (Mizuseki et al., 2009) and, accordingly, large amplitude gamma oscillations in PP-IC and Im-IC in our recordings were found shifted $180^{\circ}$ (Figure 1f). These results support the use of multichannel recordings and source separation tools to investigate interactions between theta and gamma current generators in multiple layers of the hippocampal formation.

The relative phase and coherence of these components was first compared with the theta oscillation recorded at the pyramidal layer of CA1, commonly used as the reference for temporal interactions in the hippocampus. We measured coherence and the inter-cycle phase clustering index (ICPC) which, in addition to a measure of coherence, computes the phase differences between signals in a cycle by cycle basis (see Supplementary Methods). All IC- 
LFPs exhibited prominent coherence with the LFP signal mainly in the theta range (and its first harmonic, Figure 1g). Similarly, the coherence at theta frequency was high between IC-LFP pairs (0.41, 0.31, 0.61, for Sch-lm, Sch-PP and lm-PP, respectively, Figure 1g), being larger between EC-associated generators ( $\mathrm{p}<0.0001$, ANOVA with degrees of freedom corrected by Greenhouse-Geisser, F(1.091, 4.363)=89.33). These results were confirmed by the ICPC analysis, showing significant coupling in the theta range with the reference LFP signal (ICPC $=0.50 / 0.73 / 0.61$ for Sch-IC/lm-IC/PP-IC vs. CA1 LFP, respectively, $\mathrm{p}<0.0001$, surrogate test), which also showed the characteristic phase shift across layers $(\pi / 2,0.8 \pi$ and $1.1 \pi$ radians for Sch-IC, lm-IC and PP-IC, respectively; Figure 1h). Interestingly, the lack of coherence closer to the unit between theta oscillations in the IC-LFPs was already suggesting the coexistence of different theta current generators with certain degree of independency, rather than the artificial breakdown of a unique theta rhythm into spatially segregated components. In the latter, the coherence between the oscillations should be maximum, as they would be three components of one single wave.

a

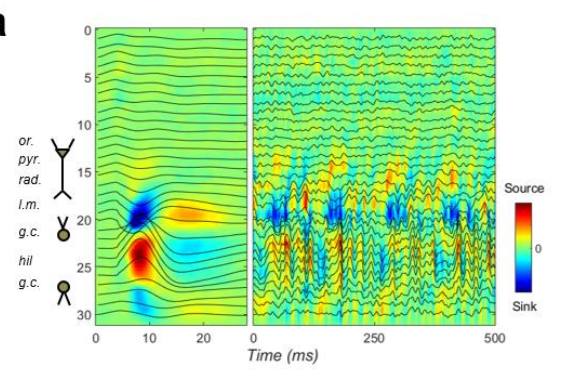

b

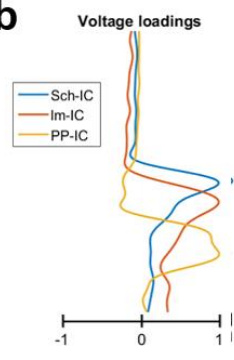

e

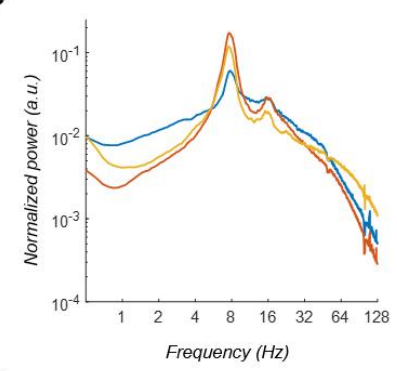

h

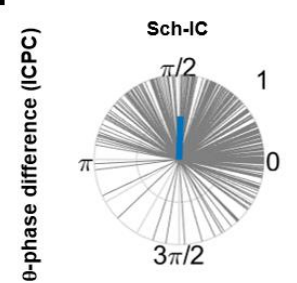

f
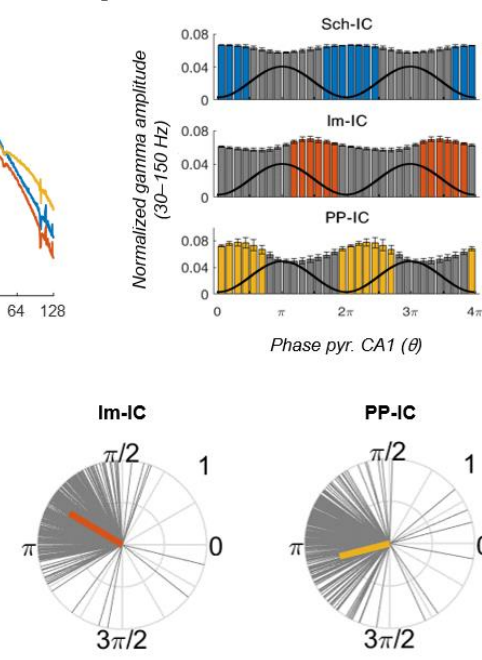

Phase pyr. CA1 ( $\theta)$

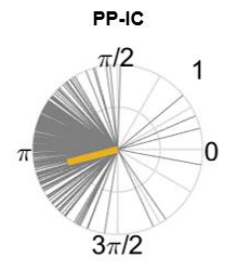

$3 \pi / 2$

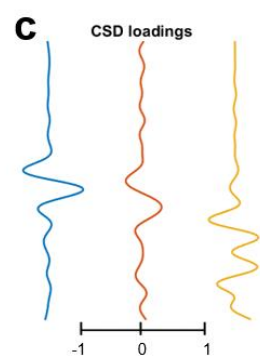

g
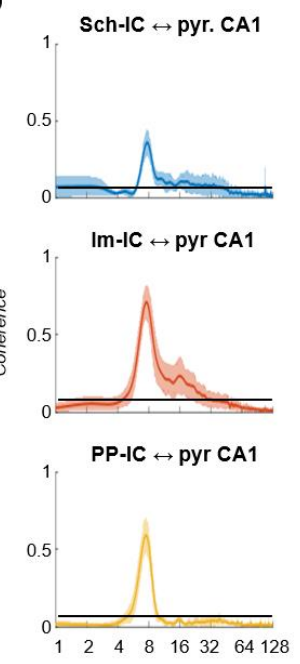

Frequency $(\mathrm{Hz})$

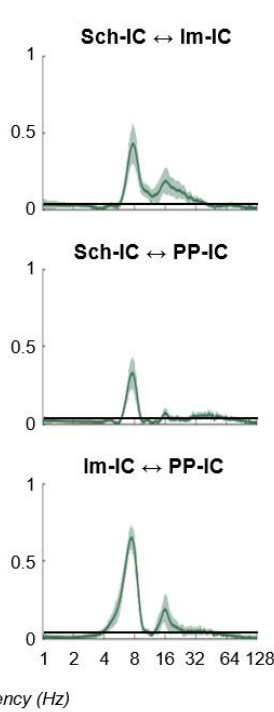

$\mathrm{Im}-\mathrm{IC} \leftrightarrow \mathrm{PP}-\mathrm{IC}$

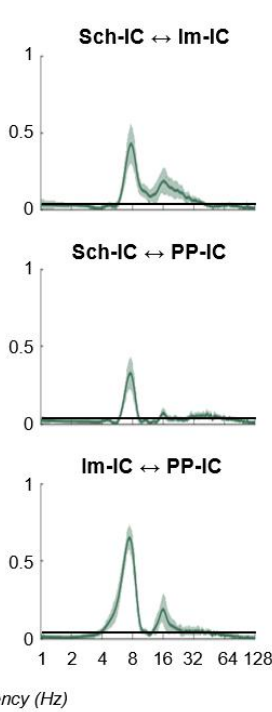

d

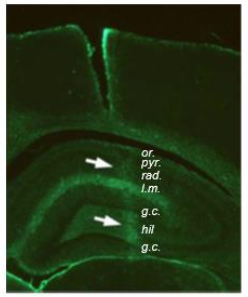

Figure 1. Pathway-specific local field potentials (LFPs). 
(a) Depth profiles of the electrophysiological signals recorded in the dorsal hippocampus (32 recordings, sites spaced every $100 \mu \mathrm{m}$ ) evoked by an electric pulse stimulating the perforant pathway (left panel) or during resting activity (right panel). Black traces represent the LFPs and color maps the corresponding current source density (CSD). Evoked activity was used to consistently localize the electrodes during implantation. Or, stratum oriens; pyr, pyramidal layer; rad, stratum radiatum; Im, stratum lacunosum-moleculare; gc, granule cell layer; hil, hilus.

( $b$ and c) Examples of voltage- and CSD-loadings of the three pathway-specific LFPs extracted with the ICA, with maximum loadings overlapping the corresponding afferent layers in the str. radiatum (SchIC), lacunosum-moleculare (Im-IC) and the molecular layer of the dentate gyrus (PP-IC).

(d) Position of the recording electrode in one representative animal (arrows). The histological section is immunostained with GFAP antibodies.

(e) The power spectra of the three ICs averaged across subjects show a clear peak at the theta frequency and a broadband gamma activity.

(f) Distribution of gamma amplitude (mean \pm s.e.m.) in IC-LFPs along the theta cycles of the LFP recorded in pyr CA1, where its trough and peak coincide with 0 and $\pi$ radians, respectively. Black waves are an example of the theta oscillation. Colorcoded bars represent statistically significant values relative to the surrogated distribution in all animals (Supplementary Methods).

(g) Coherence analysis comparing the IC-LFPs against the LFP recorded in pyr CA1 (left) and across them (right). Black lines represent the statistical threshold.

(h) Theta phase difference between IC-LFPs and LFPs recorded in CA1 pyr layer. Gray lines represent individual theta wave's phase relative to CA1 pyr theta trough (at $0 / 2 \pi$ radians). Average phase is represented by the colored thick line. Length of the thick line represents ICPC.

See also Figures S1, S2, and S3.

\section{Different theta frameworks coexist in the dorsal hippocampus}

To provide direct evidence of the independency between theta current generators, we next used an optogenetic approach (Figure 2). Using a transgenic rat cre line (LE-TG[PvalbiCre]2Ottc, NIDA, USA) and adeno-associated virus (AAV1-EF1a-DIO-hChR2(H134R)eYFP-WPRE-hGH, Penn Vector Core, USA) injected in the dorsal CA3, we expressed the excitatory Channelrhodopsin-2 (ChR2) in parvalbumin positive (PV+) interneurons (Figure 2a, Supplementary Methods Elena). Animals $(n=3)$ implanted bilaterally with optic fibres targeting dorsal CA3, and multichannel electrophysiological recordings as before (Figure 1) were used to test the hypothesis that hippocampal theta generators can be modulated independently by activating CA3 PV-interneurons and decreasing the Schaffer collateral output. As shown in Figure 2c, blue light illumination $(460 \mathrm{~nm})$ in animals freely-exploring an open field, significantly and specifically decreased the power of theta in the Sch-IC and the corresponding pathway-specific low gamma oscillations. In contrast, the oscillatory activities (power and peak frequency) in Im-IC and PP-IC were preserved (Figure 2c). The modulation of theta power specifically in the Sch-IC with the preservation of peak theta frequencies in the 
three generators, supported the existence of different theta oscillators in CA3 and EC (Kocsis et al., 1999).

We next investigated in more detail the functional interactions between the three ICLFP theta frequencies. To reduce the error of the theta phase estimation to less than $1 \%$ of the theta cycle, we selected for further analysis only those epochs in which theta power was four times higher than delta (1-4 Hz) activity (Supplementary Methods and Figure S2 for a mathematical validation of this threshold). As expected from the results in figure $1 \mathrm{~g}$ and $1 \mathrm{~h}$, theta interactions between pathways were not constant in time, but presented periods of high and low synchronization (Figure 2d). To get insight into these states, we computed a dynamic ICPC for each theta cycle, measuring the variation of the phase relationship between theta oscillations with respect to the previous and consecutive cycles (Supplementary Methods, Figure S3). The dynamic ICPC was measured for all pairs of IC-LFPs and for the three signals simultaneously (Figure 2e, Figure S4). The distribution of the ICPC over all animals showed a peak close to perfect phase locking, but with an important tail of low-synchronized epochs, with approximately the $20 \%$ of the cycles presenting an ICPC lower than 0.8. Again, the highest synchronization was found between PP-IC and lm-IC, in agreement with the coherence analyses (Figure 1g) and consistent with the origin of these generators in two sublayers of the same cortical regions (EC). In order to understand better the temporal dynamics of the theta couplings, we analysed the variability of the ICPC across time by comparing the value of a given cycle to that of the previous ones (Figure 2f). These results provided a monotonously rising curve up to $0.75 \mathrm{~s}$; from there on, the curve hardly increased. This indicates that the coupling strength between consecutive cycles spreads on a time scale in the order of one second, thus expecting dynamical changes in the ICPC in this time scale. Overall, this methodology allowed a cycle-by-cycle characterization of the temporal synchronization in the network, highlighting dynamical changes in the coupling strength between hippocampal pathways in the theta range. 
a
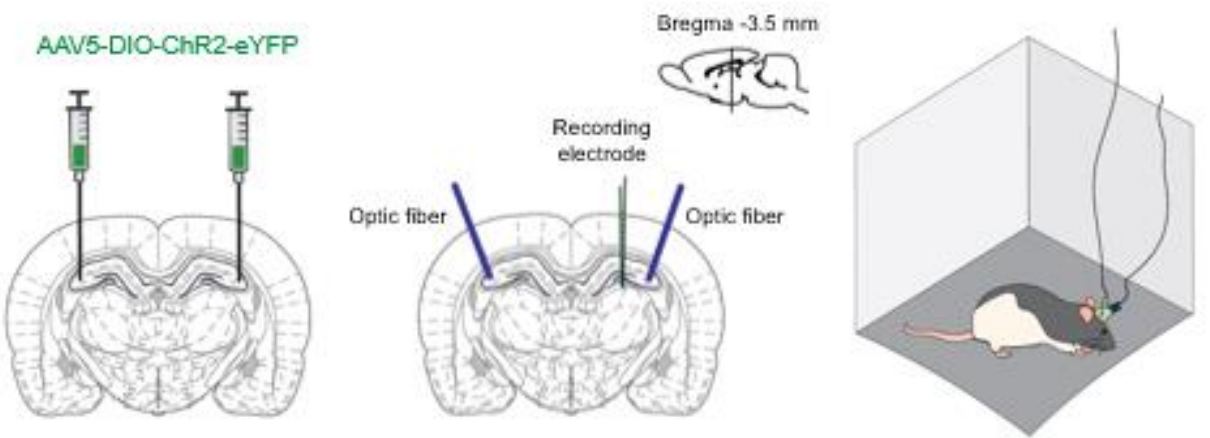

b
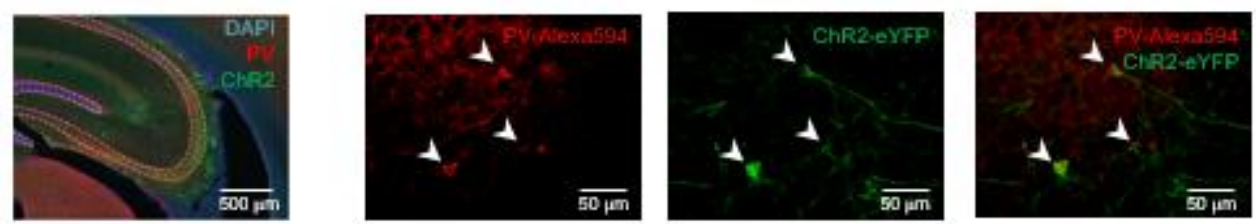

C
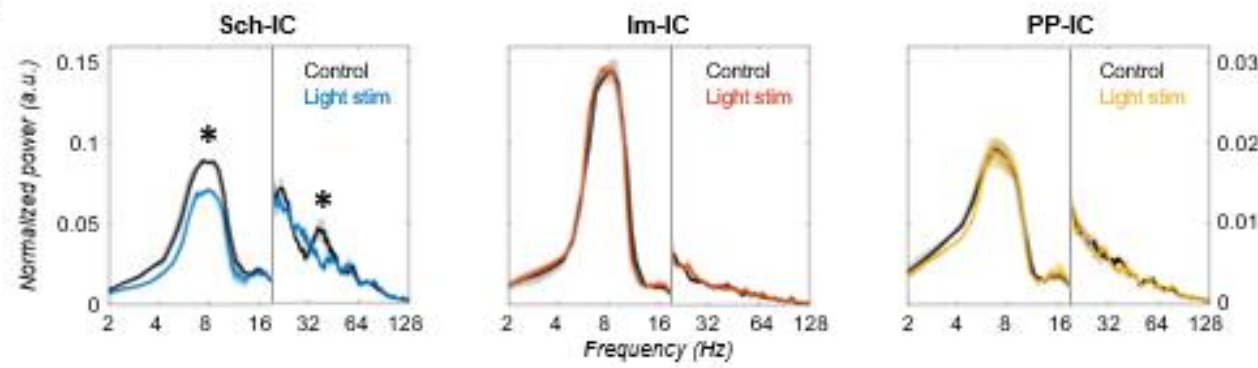

d
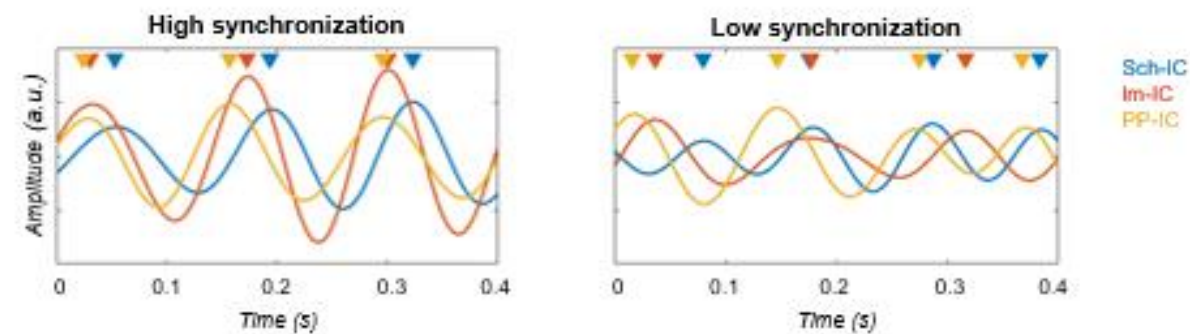

Im-IC

$P P, I C$

e
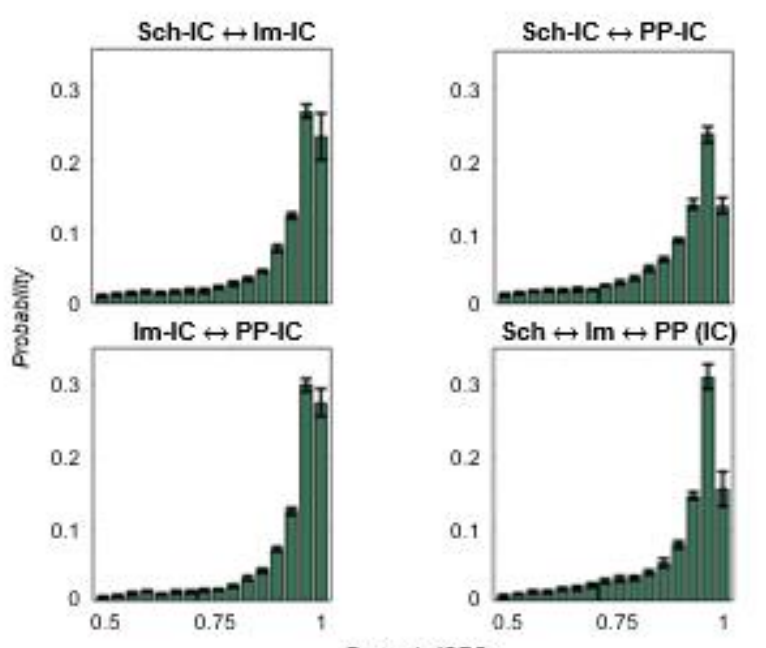

f

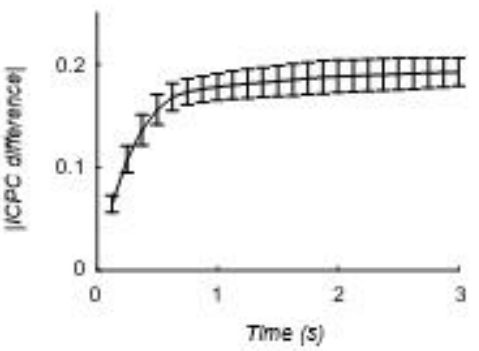

Figure 2. Different theta frameworks coexist in the dorsal hippocampus. 
(a) Schematic representation of virus injections (left), implantation of optic fibers and multichannel electrode (middle) and experimental setup (right).

(b) Representative images of coronal sections confirming the specificity of ChR2 infection in PV interneurons in dorsal CA3. Left, low magnification image showing overlapped DAPI staining (blue), $\mathrm{PV}+$ immunostaining (red) and ChR2-eYFP expression (green). Right, higher magnification images from the CA3 region showing PV+ immunostaining (red), ChR2-eYFP expression (green) and their colocalization. White arrows point to double PV+ and eYFP+ interneurons.

(c) Optogenetic manipulation of PV interneurons. Power spectra analysis (mean \pm s.e.m) of the IC-LFPs during light OFF (black traces) and light ON (colored traces) conditions. Blue light $(470 \mathrm{~nm}$ ) illumination reduced theta and slow-gamma power selectively in Sch-IC $\left({ }^{*} p<0.05\right.$, paired $t$-test, $t=7.88 / 7.34$ for theta/gamma Sch-IC, $\mathrm{N}=3)$. Note different $\mathrm{y}$-axis scales for low $(<20 \mathrm{~Hz})$ and high $(>20 \mathrm{~Hz})$ frequencies for visualization purposes.

(d) Traces of theta-filtered IC-LFPs showing epochs with high (top) and low (bottom) phase locking between their rhythms. Triangles pointing to the peak of theta cycles in each IC-LFP (differentiated by colors as before) are used to highlight variability in phase differences.

(e) Distribution of ICPC values per theta cycle between pairs of components or with the three IC-LFPs simultaneously (mean \pm s.e.m).

See also Figure S4.

(f) Dynamics of ICPC changes. The y axis represents the average ICPC differences in absolute values between two cycles, and the $\mathrm{x}$ axis represents the time difference between their occurrences. Consecutive cycles demonstrate more similar ICPC values than those separated in time up to $0.75 \mathrm{~s}$.

\section{Theta-gamma CFC reflects pathway-specific interactions}

The above results supported the coexistence of different temporal frames in the theta range to organize hippocampal activity. Thus, since gamma activity is nested to the theta cycle, it opened the possibility to multiple theta-gamma interactions (Figure 3a). A conventional analysis of theta-gamma CFC (Figure 3b) using as a phase reference the theta in the LFP recorded in the pyramidal layer, as is usually done (Colgin, 2015a; Colgin et al., 2009; Csicsvari et al., 1999; Fernández-Ruiz et al., 2017; Lasztóczi and Klausberger, 2016, 2014; Schomburg et al., 2014; Tort et al., 2009, 2008), identified the typical coupling between CA1 theta and a slow gamma band of CA3 origin (Sch-IC; maximal modulation at $37.5 \pm 5 \mathrm{~Hz}$, CA1 $\gamma$ S) (Colgin et al., 2009; Lasztóczi and Klausberger, 2014; Schomburg et al., 2014) and a medium gamma band of EC3 origin (lm-IC; $82.5 \pm 4 \mathrm{~Hz}$ medium gamma, CA1 $\gamma \mathrm{M})$ (Colgin et al., 2009; Lasztóczi and Klausberger, 2014; Schomburg et al., 2014). The analysis also revealed an additional theta-nested fast gamma band $(130 \pm 10 \mathrm{~Hz}$, ) in the mid-molecular layer of the $\mathrm{DG}(\mathrm{DG} \gamma \mathrm{F})$ overlapping the terminal field of EC2 inputs, compatible with the previously found theta-gamma CFC in the DG (Bragin et al., 1995). Interestingly, when we then computed the CFC using as references the different theta oscillations separated in the IC-LFPs, we found stronger CFC as demonstrated by the significantly higher modulation indices (MI; Figure 3b). The result was not totally unexpected, since we had found that the theta oscillation recorded in 
the LFP and typically used as a reference in CFC analysis, was indeed a mixture of different theta generators of variable coherence (see Figure 2d-e above).

We finally asked whether pathway specific gamma activities were preferentially coupled to the theta oscillation in their same afferent pathway, likely reflecting local computations, or in different pathways, thus reflecting inter-pathway interactions, or both. Results in Figure 3c-d demonstrate a dominant CFC between oscillations recorded in the same IC-LFP. Therefore, theta-gamma CFC mainly reflects pathway-specific interactions, rather than a unique carrier theta wave to which the gamma activity from different origins is multiplexed in segregated theta-gamma channels. The higher theta-gamma CFC found by using pathway-specific theta references, provided yet another indication of the coexistence and relevance of distinct temporal theta frameworks in the hippocampus.

a

b
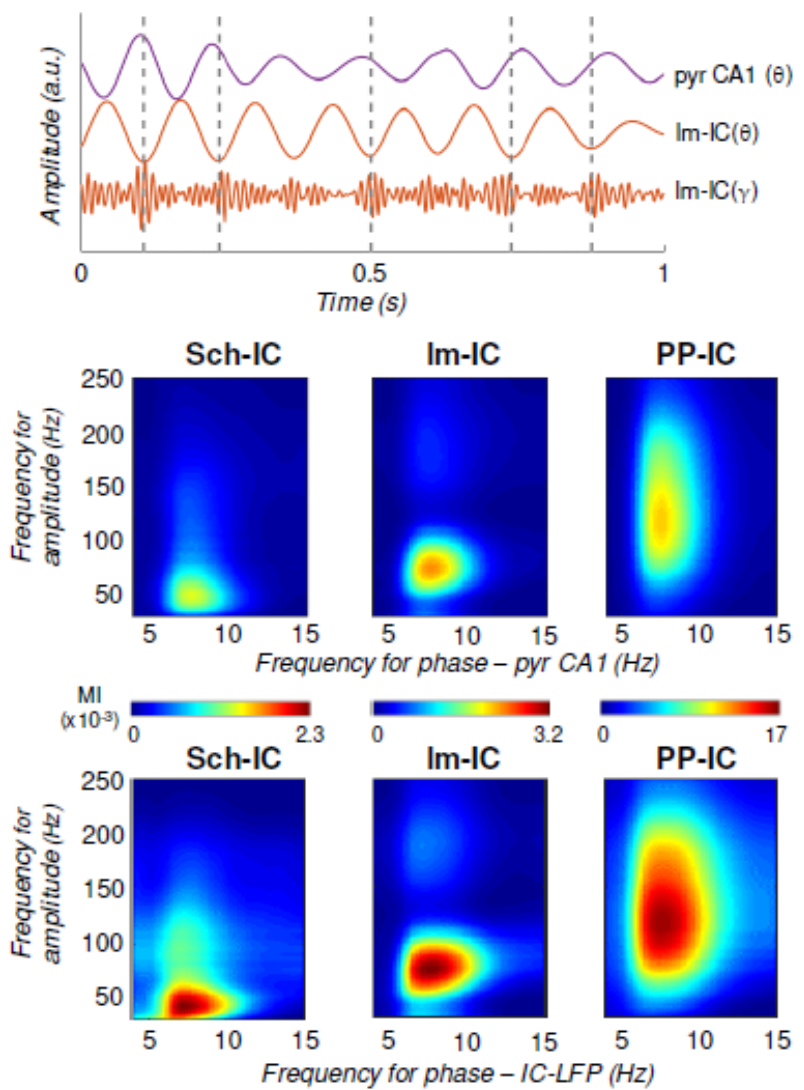

C
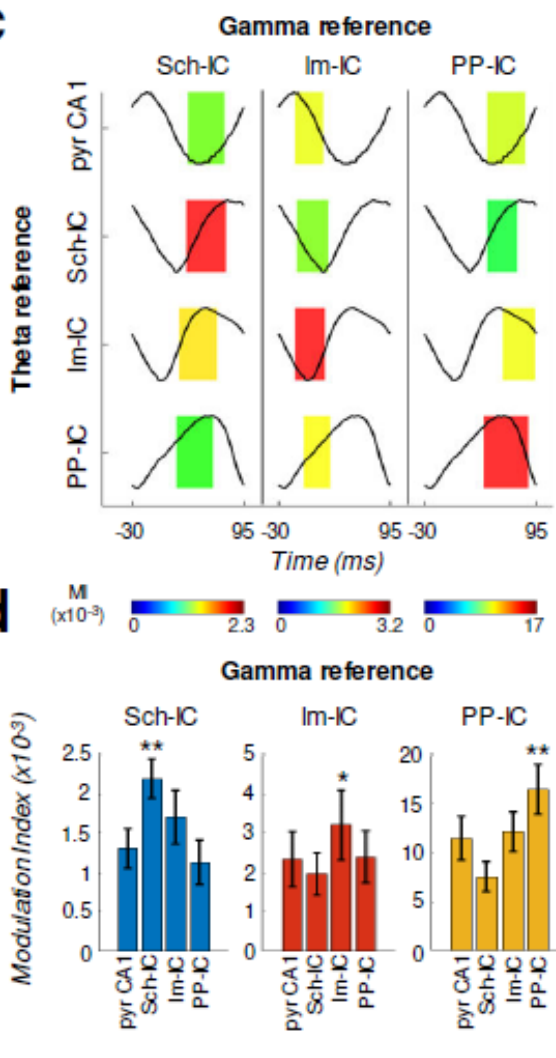

Theta reference

Figure 3. Theta-gamma coupling reflects pathway-specific interactions.

(a) Representative theta- and gamma-filtered traces of Im-IC showing how the gamma envelope is phase-locked at the trough of the theta oscillation recorded in the same IC-LFP, but not with the oscillation recorded in the CA1 pyramidal layer (pyr CA1).

(b) Modulation strength (color coded $\mathrm{MI}$ ) of gamma amplitude $(30-250 \mathrm{~Hz})$ in the IC-LFPs and the theta phase recorded in the pyr CA1 LFP (upper panels) or the pathway-specific thetas in the corresponding IC-LFPs (lower panels). 
(c) Interregional CFC across all gamma and theta oscillations recorded in the three IC-LFPs and pyr CA1. Each rectangle represents the MI between the theta phase and the gamma amplitude at a single specific frequency (gamma reference: slow/medium/fast gamma for Sch-IC/Im-IC/PP-IC). The location and width of the rectangles indicate the theta phase at which gamma amplitude is coupled, and the color the MI (color scales at the bottom of each column). Theta waveforms (black traces) are extracted as the average of all theta cycles in the corresponding signals. The highest CFC strength (red rectangle) was always found between theta and gamma oscillations of the same pathway.

(d) Maximum theta-gamma CFC corresponds to oscillations recorded in the same IC-LFP, higher than any between-pathway combination. Left/middle/right panel represents the $\mathrm{MI}$ (mean \pm s.e.m.) between slow/medium/fast gamma recorded from Sch-IC/Im-IC/PP-IC, and the theta phase of all ICLFPs and the LFP from pyrCA1. Significantly stronger MI values were found in all cases when theta phases were calculated from the corresponding pathway-specific generators, in contrast to pyr CA1 LFP, and when theta and gamma oscillations had the same origin $(* / * * p<0.05 / 0.01$, one-way ANOVA of repeated measurements between $\mathrm{MI}$ with the same theta reference, followed by Bonferroni correction, $F(1.182,4.729)=36.16 / F(1.133,4.531)=8.649 / F(1.555,6.219)=35.32$ for Sch-IC/Im-IC/PPIC as theta reference, $\mathrm{N}=5$ ).

\section{High theta-gamma CFC is associated to synchronization between theta frameworks}

Having shown that theta generators can be modulated independently and present variable synchrony (Figure 2) and gamma nesting is pathway-specific (Figure 3), we next explored the theta and gamma features accounting for the different synchronization states. We found that theta power in all IC-LFPs correlated with the ICPC, with larger theta power associated with states of higher synchronization (Figure $4 \mathrm{a}, 4 \mathrm{~b}$ and $4 \mathrm{c}$ ). Theta frequencies in Im-IC and PP-IC progressively increased with the synchronization states (Figure 4a) correlating with ICPC (Figure $4 \mathrm{~b}$ and $4 \mathrm{c}$ ). Regarding gamma activity, broadband power did not correlate with theta synchronization (not shown), in contrast to narrowband power (slow/medium/fast gamma for Sch-IC/lm-IC/PP-IC, respectively), which correlated with the ICPC in Im-IC, but not in Sch-IC nor PP-IC (Figure 4b).

Because running speed also correlates with hippocampal theta power and frequency (Vanderwolf, 1969), we performed a multiple linear regression (MLR) analysis including running speed, theta power and theta frequency as explanatory variables to predict the ICPC (Figure 4c). This analysis allowed us to estimate the contribution of each variable to the ICPC that cannot be accounted by any other variable in the model. We used for the analysis all theta cycles recorded while animals were exploring a familiar open-field. The MI was not included in the MLR since its value for individual theta cycles is not reliable. The result demonstrated the main effect of theta power on the ICPC value, with a lower, but significant, contribution of theta frequency and running speed (Figure $4 \mathrm{c}, \mathrm{p}<0.05$, t-test against zero between beta values of each factor, Bonferroni corrected, Supplementary Methods). 
Finally, this analysis unveiled a striking correlation between the CFC and theta synchronization (Figure $4 \mathrm{~b}$ and $4 \mathrm{~d}$ ). Strong theta-gamma modulation was associated with high ICPC values, while weak or nearly absent CFC was found in periods of low synchronization. Note that, as mentioned above, only cycles with high theta power activity were selected in this analysis (Figure 4d), so that signal's power could not affect the estimation of its phase (Figure S2), thus preventing the introduction of any bias in the synchronization measurement (Figure S2, Supplementary Methods). This result indicated that within-pathway CFC was associated to the synchronization between pathways. 
a
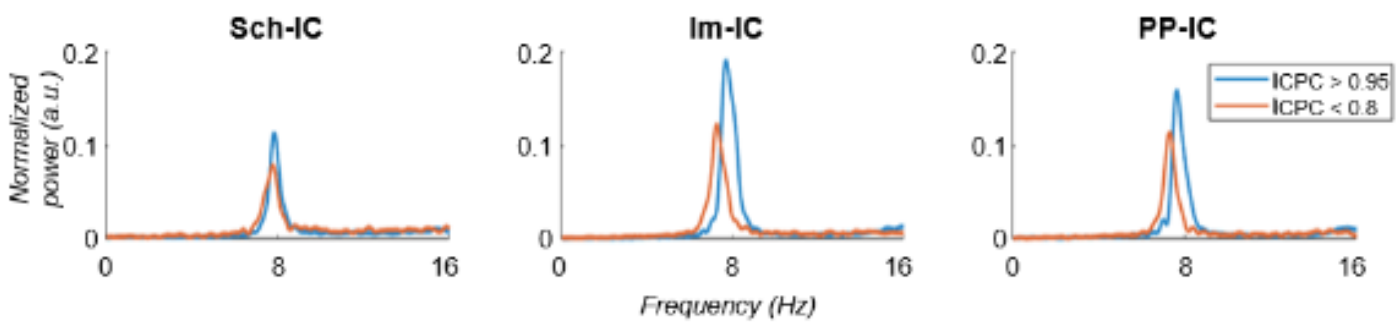

b

Sch-IC

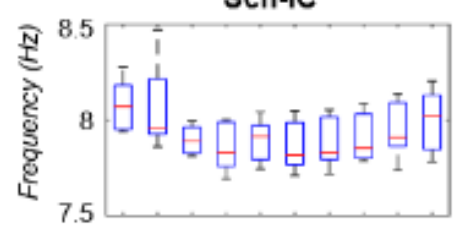

Im-IC

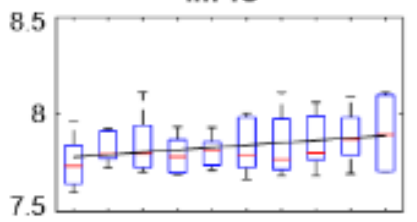

PP-IC
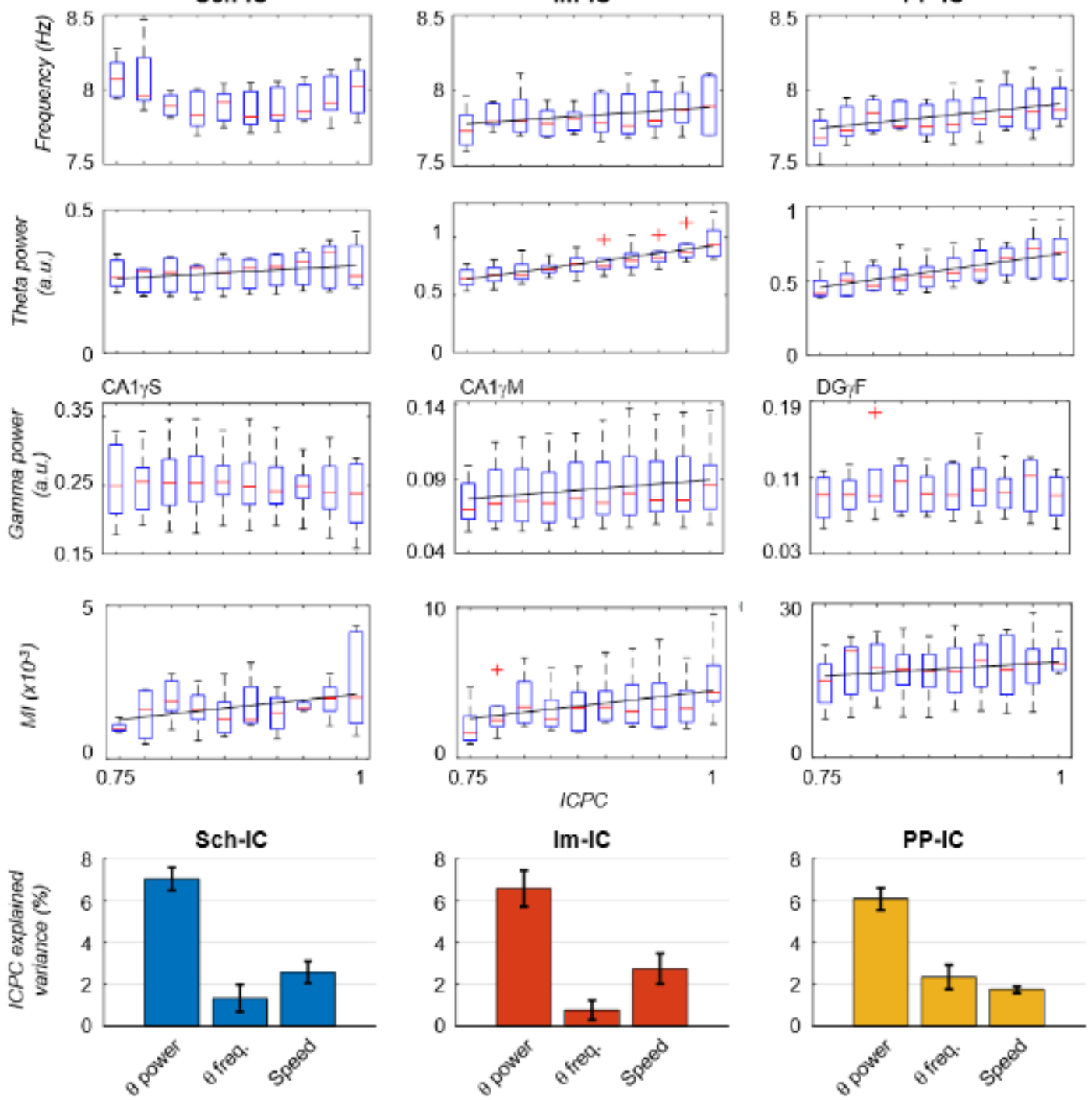

d

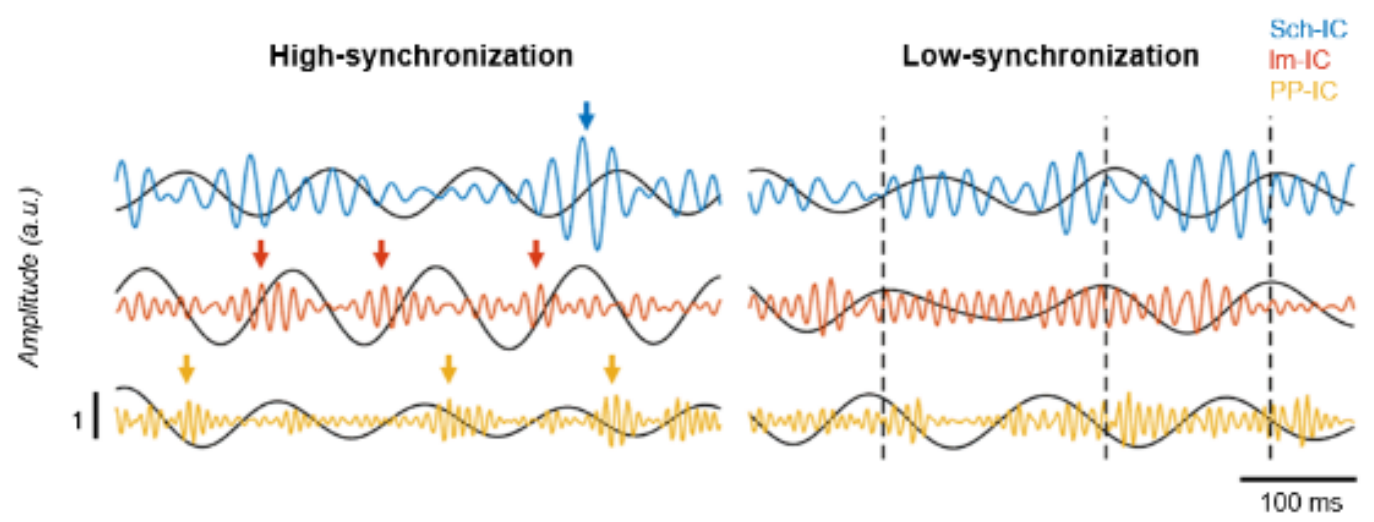


Figure 4. Characterization of theta oscillations as a function of their synchronization.

(a) Power spectrum of the IC-LFPs during high (blue, ICPC $>0.95$ ) and low (red, ICPC $<0.8$ ) theta synchronization epochs. A strong increase of the theta peak can be seen in all IC-LFPs during theta synchronization, together with a right-shift of the peak frequency for Im-IC and PP-IC.

(b) Theta frequency correlated with ICPC in Im-IC and PP-IC (black lines represent statistically significant linear correlations; $\mathrm{R}=0.94 / 0.92, \mathrm{p}<0.05$, respectively, surrogate test). Theta power correlated in all ICs with the synchronization state $(R=0.93 / 0.99 / 0.99, p<0.05 / 0.0001 / 0.0001$, respectively). Medium-gamma band was correlated with Im-IC $(R=0.96, p<0.0001)$, but not with SchIC nor PP-IC. CFC in all pathway-specific generators increased with ICPC ( $R=0.71 / 0.82 / 0.77$, $p<0.01 / 0.05 / 0.001$, respectively). Correlations were computed on the mean values of each ICPC bin. For all figures, the central mark of the box indicates the median, and the bottom and top edges of the box indicate the 25th and 75th percentiles, respectively. The whiskers extend to the most extreme data points not considered outliers, and the outliers are plotted individually with red asterisks.

(c) MLR analysis including theta power, theta frequency and speed as factors to predict the ICPC. Bars represents the variance explained by each factor that cannot be accounted for by other variables. For all cases, the contribution was considered significant (see Supplementary Methods)

(d) Representative theta and gamma traces showing differences in CFC and theta frequency in two synchronization states, from recordings with comparable theta power.

\section{Gamma oscillations consistently precede theta waves}

Theta and gamma oscillations reflect the extracellularly added excitatory and inhibitory synaptic and active dendritic currents of two processes occurring at different timescales (Herreras, 2016). We hypothesize that CFC may reflect a mechanism through which fast excitation-inhibition interactions organize the activity of principal cells in different theta frameworks found in our analysis. We then looked for an indication of directionality in the interaction between the two frequencies, and computed the cross-frequency directionality index (CFD) (Jiang et al., 2015), based on the phase-slope index to compute the phase difference between two signals. This methodology was specially developed to estimate the directionality between signals with large differences in signal to noise ratio, as theta and gamma frequencies, demonstrating in these conditions to be more efficient than classical approaches such as Granger Causality (Granger, 1969; Jiang et al., 2015; Nolte et al., 2010). In CFD, an increase of the phase difference between the theta phase and the gamma amplitude with frequency gives rise to a positive slope of the phase spectrum (i.e. a positive CFD value) when the phase of the slow oscillation consistently precedes the amplitude of the fast, this is, when the time difference between a theta phase and the next burst of gamma activity is constant. The slope is negative when the amplitude of the fast oscillation consistently precedes the phase of the slow, in our analysis, when the delay from the gamma activity to the next theta cycle is constant. As shown in Figure 5a for the group data, and Figure S5 for individual animals, CFD resulted in negative values (amplitude-phase coupling, APC) for the specific 
gamma bands nested to the theta oscillations in the corresponding IC-LFPs. This gamma amplitude to theta phase directionality is given by the consistent anticipation of the gamma activity to the theta phase. In Figure 5b, there is a representative example of the gamma-totheta directionality in PP-IC. The delay from gamma to theta is almost fixed, while in the opposite direction (theta to gamma) is highly variable.

To validate the finding, we also computed the CFD directly in the LFP signals recorded in the different hippocampal layers. To compare with the IC-LFPs, we chose the LFP signals from the channels matching the site of maximum contribution to each IC-LFP (Figure 5c and 5d). Negative values of CFD were found in the LFPs in str. lacunosum-moleculare and in the DG, supporting the driving role of gamma oscillations over the phase of the theta waves. We could not find a significant directionality in the case of the str. radiatum LFP and, in all cases, the absolute value of the CFD was higher using IC-LFPs than LFPs (Figure 5d), demonstrating that source-separation tools outperform the use of raw LFPs to investigate pathway interactions. Overall, our CFD analysis suggests that the neuronal circuits supporting gamma oscillations in the hippocampus set the timing of principal cells activity in the theta range, as reflected in the phase of the recorded theta oscillations.

Finally, an interesting added value of CFD is to discard potential contributions of harmonics to the CFC (Sheremet et al., 2019, 2016; Zhou et al., 2019). Spurious couplings between the fundamental $8 \mathrm{~Hz}$ frequency of the theta rhythm and its harmonics, i.e. at $16 \mathrm{~Hz}$ or $32 \mathrm{~Hz}$, could be in principle possible (Aru et al., 2015). However, in this scenario, both the theta and gamma oscillations are components of the same signal and would be perfectly coupled. Therefore, the CFD analysis would fail in detecting a directionality between them. The presence of a significant nonzero CFD (either positive or negative) demonstrates that a found CFC is not just originated by the presence of harmonics, while a zero value would not allow to discriminate the nature of the CFC. In our analysis, the found significant negative values of CFD align with the first case. 
a
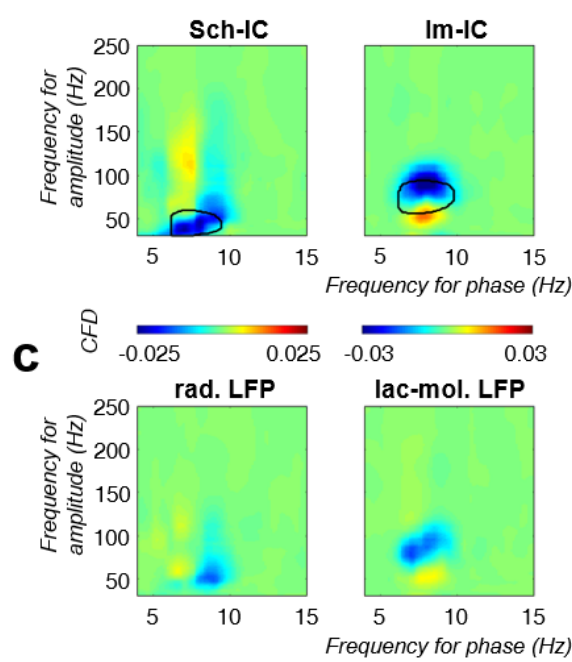

b
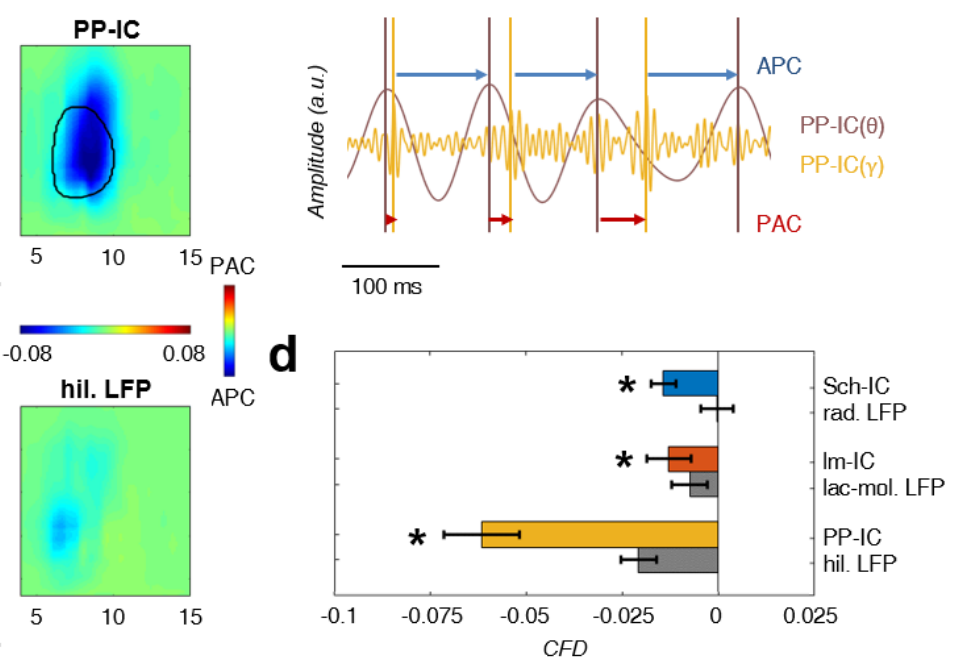

Figure 5. CFD analysis reveals that gamma activity modulates the phase of theta.

(a) CFD analysis of the pathway-specific signals demonstrates maximum negative values (APC) for those pairs of theta-gamma oscillations with the highest CFC (encircled area). These results suggested that gamma oscillations modulate the theta phase.

(b) Example of gamma-to-theta coupling in the PP-IC. The time difference from the maximum of gamma activity to the theta peak is almost fixed (APC, blue arrows), while the distance from theta to gamma varies in each cycle (PAC, red arrows).

(c) Same CFD analysis as in (a) using the raw LFP from different hippocampal layers confirmed the APC directionality.

(d) Comparison between CFD values using IC-LFPs and LFPs showed convergent results, with IC-LFPs outperforming the raw signals ( ${ }^{*} p<0.05$, paired $t$-test across subjects between IC and LFP values for each IC-LFP separately, $t=3.99 / 2.98 / 4.59$ for Sch-IC/Im-IC/PP-IC, $N=5)$.

See also Figure $\mathrm{S} 5$.

\section{Behavioural modulation of theta-gamma CFC and theta synchronization}

Previous studies have shown that both CFC and inter-region coherence, independently, correlate with learning (Canolty et al., 2006; Engel et al., 2001; Fries, 2015, 2005; Palva et al., 2005; Tort et al., 2009, 2008). Our analysis (Figure 4) now showed that both phenomena seem to be linked. Therefore, in our final set of experiments, we looked for behavioral evidence in support of the hypothesis that they are part of a common mechanism to flexibly integrate or segregate neuronal computations. More specifically, we hypothesized that layer-specific interaction will phase-lock theta oscillations between layers to facilitate the integration in CA1 of CA3-mediated and EC-mediated information streams, for instance, in learning conditions requiring the comparison of context representations from memory (Sch-IC pathway) and from the environment (lm-IC and PP-IC pathways) (Buzsáki and Moser, 2013; Dudai and Morris, 2013; Wang and Morris, 2010). 
One such learning conditions is mismatch novelty (Lever et al., 2006) in which the subject is re-exposed to a previously visited context which has been modified. The 'mismatch' occurs when comparing the expected representation from memory and the found representation in the environment. To test whether ICPC and CFC increase in parallel during mismatch novelty, we trained the animals in a task in which, after habituation to an open field ( $8 \mathrm{~min}$ session 1 per day during 8-10 days, Figure 6a control), we introduced a novel tactile stimulus in the floor of the otherwise unchanged field (Figure 6a novelty, see Supplementary Methods). We computed and compared theta synchrony and CFC between the novelty session and the habituation session the day before. When the animal entered the arena, the ICPC between theta oscillations was high and comparable in both conditions during the first two minutes of exploration (Figure $6 \mathrm{~b}, \mathrm{t} 1$ ). As the animal explored the context, synchronization remained high during novelty, but rapidly decayed in the known environment (Figure 6b, t2). Consistent with the notion of information transmission to update an existing memory, by the end of the exploration time both conditions decreased to the same level of theta synchronization (Figure $6 \mathrm{~b}, \mathrm{t} 3)$, when the introduced tactile stimulus lost its novelty. As a control, we tested locomotor activity comparing movement velocity between novelty and habituation sessions (Figure 6c), without finding differences between sessions ( $p>0.3$, t-test (Figure 6c). Therefore, differences in the ICPC cannot be solely explained by changes in locomotor activity. We used MLR analysis as before, to investigate now the independent contribution of theta power and frequency, experimental condition (control vs. novelty sessions), running speed and time in the task to the measured ICPC (Figure 6d). We found that theta power and the experimental condition are the main factors contributing to the ICPC value with other variables such us running speed and time marginally contributing.

We then computed the CFC index (MI) in the same recordings and found that it paralleled the changes in theta synchronization during the complete session in both conditions, as shown in Figure $6 \mathrm{~b}$ and $6 \mathrm{e}$. The CFC strength was higher in the three IC-LFPs during the novelty sessions associated with the higher theta synchronization, and decreased towards the end of the session in parallel with the ICPC (Figure 6e). In contrast to mismatch novelty, uncharted novelty involves the exposure to a previously unvisited context and therefore it lacks a memory representation. Interestingly, a recent study using an uncharted novelty test showed increased theta-gamma CFC exclusively in EC pathways, but not in the CA3 $\rightarrow$ CA1 pathway (Barth et al., 2018). Thus, in the absence of a memory representation, only EC-pathways 
conveying information about the environmental cues demonstrate enhanced theta-gamma coupling, lending support to our hypothesis.

Furthermore, in the above uncharted novelty study, the authors showed that CFC in EC pathways preferentially occurs when the animal is rearing on its hind legs, an exploratory response to novelty (Lever et al., 2006), which is also associated with increased theta frequency (Barth et al., 2018). To investigate the potential contribution of rearing behaviour to our findings in the mismatch novelty task, we removed from our recordings the epochs in which animals were rearing on their hind legs and then reanalysed ICPC and CFC. As shown in Figure S7 the increased theta synchronization between the three IC-LFPs is maintained during novelty in the absence of rearing epochs. Similarly, the MI is higher during novelty, although more variable, likely reflecting to the decrease in the number of data samples after rearing removal. Importantly, increased CFC reached significance in the rearing-associated EC pathways (Barth et al., 2018). Overall, the changes found in mismatch novelty cannot be solely explained by the rearing behaviour. Furthermore, we measured theta frequency in the complete time series and compared it between control and novelty conditions, and did not find an increase comparable to unchart noverty (Barth et al., 2018), but rather a significant decrease for Sch-IC and PP-IC theta frequency in 22 (Figure S6) (Wells et al., 2013). 
a

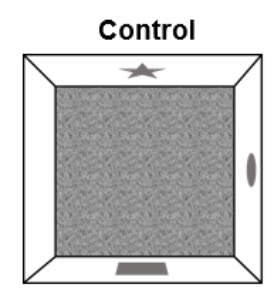

C
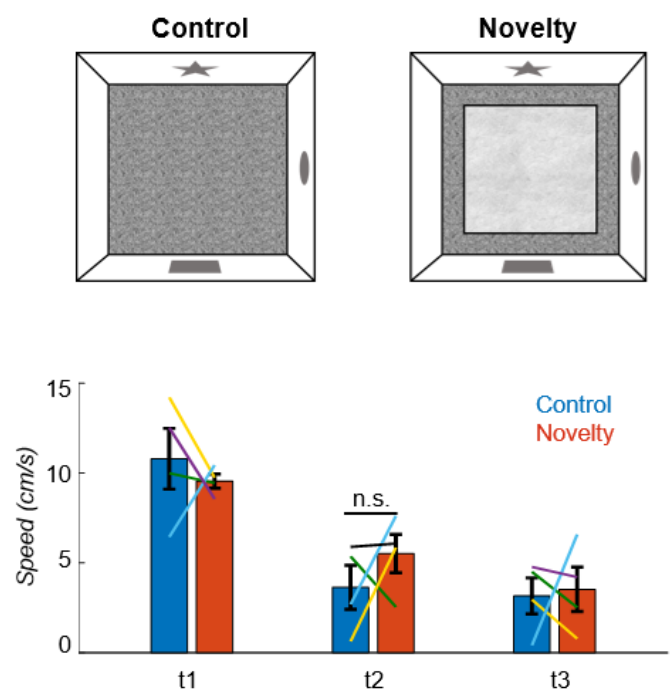

b

d
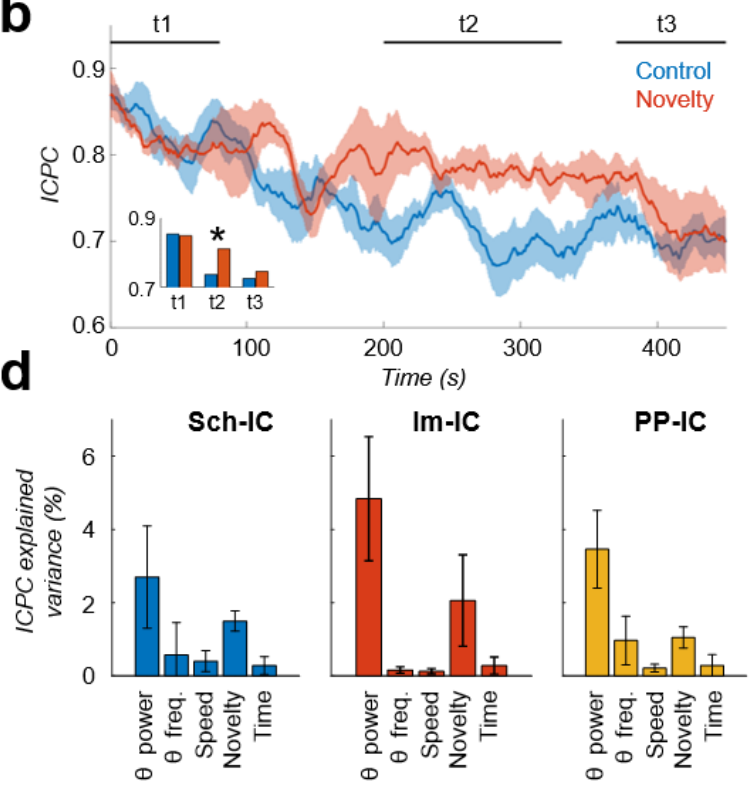

e
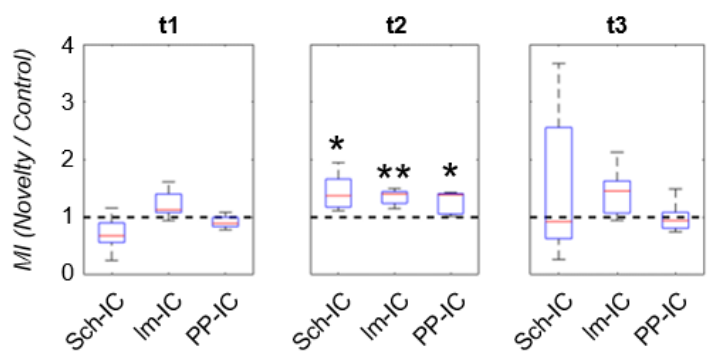

Figure 6: Local theta-gamma CFC and theta synchronization increase in parallel during mismatch novelty.

(a) Scheme of known (left) and novel (right) open-field contexts. After the habituation period (control), the animals were exposed to a different floor (sand paper) located inside the familiar open field, providing a new tactile stimulus (novelty).

(b) Time evolution of the dynamic ICPC (mean \pm s.e.m. across all subjects) during exploration: before (blue) and after (red) the introduction of the novel tactile stimulus. Both conditions have a maximum ICPC value at the beginning of the task (t1), corresponding to the initial exploration, followed by a decay in control but not in novelty ( $\mathrm{t} 2$, inset $* \mathrm{p}<0.05$, paired $\mathrm{t}$-test comparing the average ICPC in control vs. novelty for each time period separately, $t=3, N=5)$. Both conditions decrease to the same ICPC level by the end of the exploration time ( $t 3)$.

(c) Averaged movement velocity of the animals during control and novelty. There were not significant differences between both conditions (paired t-test across subjects for each time period, $N=4$ ). Colourlines represent the values of each subject.

(d) MLR with theta power, theta frequency, speed, session (control or novelty) and time as independent factors contributing to ICPC. Bars represent the variance explained by each factor that cannot be accounted for by other variables. All contributions were significant (see Supplementary Methods) except for the theta frequency in Sch-IC and Im-IC.

(e) CFC ratio computed as the ratio between the averaged $\mathrm{Ml}$ in the defined time window ( $\mathrm{t} 1, \mathrm{t} 2$ and $\mathrm{t} 3)$ in the novelty condition with respect to the control one $(* \mathrm{p}<0.05$, paired t-test across subject, $\mathrm{t}=2.95 / 5.65 / 2.92$ for Sch-IC/Im-IC/PP-IC in $\mathrm{t} 2, \mathrm{~N}=5$ ).

See also Figure $\mathrm{S} 6$ and $\mathrm{S7}$ 
In a second behavioural experiment, a hippocampus-dependent delayed spatial alternation task was used in which the animal needed to remember the arm visited in the previous trial and update the memory with the choice made in the current trial (Ainge et al., 2007; Montgomery and Buzsáki, 2007; Wood et al., 2000), again relying on the interaction between context representations form memory and from external sensory cues. Rats learned in an 8-shaped T-maze to alternate between the left or right arms on successive trials for water reward (Figure 7a) until they reached performances above $80 \%$. In this task, the central arm is associated with memory recall, decision making and encoding of the current decision (DeCoteau et al., 2007; Montgomery and Buzsáki, 2007; Tort et al., 2008; Wood et al., 2000), while neuronal recordings in the side arm are thought to convey little information to predict behavioural outcomes in the following trial (Pastalkova et al., 2008; Schomburg et al., 2014). Using this task, a phase shift between theta oscillations recorded in CA1 and CA3 pyramidal layers was shown associated to the central arm (Montgomery et al., 2009). We then computed and compared theta-gamma CFC and theta synchronization in recordings obtained from the central and side arms in correct trials, selecting only those epochs were the movement velocity was comparable in both arms (Figure 7b). We found significantly increased CFC in the central arm for the three IC-LFPs (Figure 7c). This result is consistent with previously shown increased CFC in the same task in the CA1 radiatum and lacunosum-moleculare IC-LFPs (Schomburg et al., 2014). Importantly, concomitant with CFC, we also found an increase in theta ICPC in the central arm during the same epochs (Figure 7d and 7e). The results of the two behavioural tasks, thus, demonstrate that theta-gamma CFC and theta ICPC across generators are linked and preferentially occur during memory-guided exploration and mismatch novelty detection, two conditions in which internally generated memory representations need to be integrated with the incoming sensory information about external cues. They support the idea that different theta-gamma frameworks may flexibly coordinate information transmission in the hippocampus.
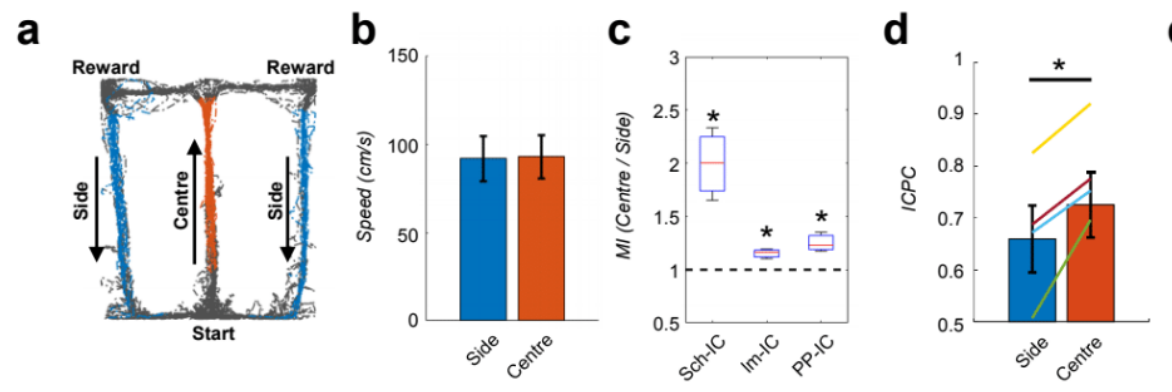

e

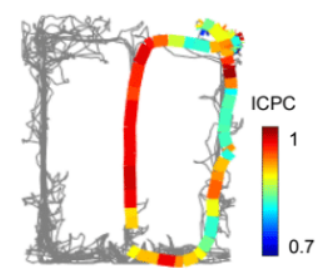


Figure 7: Local theta-gamma CFC and theta synchronization change in parallel and associated with decision making in a T-maze task.

(a) Example of running trajectories during the T-maze task.

(b) Averaged movement velocity in the centre and side arms (mean \pm s.e.m. across all subjects). Only trials with similar speed in both conditions were considered for the analysis.

(c) Ratio between the $\mathrm{Ml}$ at the centre of the maze and that at the sides $\left({ }^{*} \mathrm{p}<0.05\right.$, paired t-test across subjects, averaging all selected trials for each animal, $t=3.61 / 4.03 / 3.54$ for $\mathrm{Sch}-\mathrm{IC} / \mathrm{Im}-\mathrm{IC} / \mathrm{PP}-\mathrm{IC}, \mathrm{N}=4)$.

(d) ICPC values in the centre and side arms showing the synchronization between the three IC-LFPs (* $p<0.05$, group level paired $t$-test, $t=4.79, N=4$ ). Differences within-subject (colour lines) were done with paired t-test using all correct trials (yellow line: $p<0.0001, N=114$ trials; purple: $p<0.05, N=63$; green: $p<0.001, N=151$; blue: $\mathrm{p}=0.07, \mathrm{~N}=69$ ).

(e) Representative example of ICPC values for different locations in one trial.

\section{Discussion}

Overall, our results provide functional evidence supporting independent theta oscillations in the hippocampus whose coordination can be seen as a mechanism to channel information between hippocampal layers and bind distributed computations. Less synchronized theta states may secure relatively independent processing in local circuits of the hippocampal formation (segregation). Theta coordination (phase locking) across hippocampal layers is associated with stronger CFC. Furthermore, directionality analysis suggests that bandand pathway-specific gamma activity contributes to the synchronization of theta oscillations across layers. We thus hypothesize that the CFC reflects a mechanism operated by fast excitation-inhibition interactions to coordinate neuronal communication in theta waves. In a network with multiple connected nodes, theta-phase locking between specific nodes will further contribute to the directionality of the information flow, habilitating targets between which communication is permitted or prevented in defined time windows. We have provided evidence supporting this hypothesis by showing that $\mathrm{CFC}$ and the coordination between the theta current generators recorded in the hippocampus increases in the mnemonic process.

In this work, we have separated the LFP sources contributed by different synaptic pathways using spatial discrimination techniques based on independent component analysis (Benito et al., 2014; Fernández-Ruiz and Herreras, 2013; Herreras, 2016; Makarov et al., 2010; Makarova et al., 2011). This processing step allowed us to work with a more reliable representation of the local electrophysiological dynamics, as compared to raw LFPs or CSDs (Martín-Vázquez et al., 2013). The main drawback of LFPs is the multisource origin of the signals, a blend of dipolar (or quadrupolar) field potentials. While the CSD of multisource raw LFPs avoids the problem of volume conduction, it does not separate the co-activating current sources in the recorded region, hence the CSD of pathways targeting the same cells (e.g. CA1 
pyramidal cells) overlap and add/subtract, cancelling each other. In these conditions, the time course of the CSD is a composite one (as it is that of native LFPs) and cannot be unambiguously assigned to any of the co-activating sources. Therefore, source separation techniques are necessary to obtain the correct time course of each individual synaptic contribution.

Extensive previous research has demonstrated the existence of multiple theta rhythms and current generators in the hippocampus and EC (Buzsáki, 2002). While septal activity is required for theta rhythmicity, and lesions targeting the medial septum eliminate theta oscillation in both structures, intrinsic hippocampal activity from CA3 and extrinsic EC inputs do also contribute to the recorded theta oscillations (Buzsáki, 2002). Surgical removal of the EC unveils a theta oscillation that depends on the integrity of CA3 and is highly coherent across hippocampal layers (Bragin et al., 1995). In the presence of an intact EC, however, the coherence between theta signals in the stratum radiatum and lacunosum-moleculare is reduced (Kocsis et al., 1999), consistent with an input competition between CA3 and EC3. The different modulation of theta oscillations is further supported by our optogenetic experiment and the fact that amplitude, frequency and phase vary independently and dynamically in the theta generators (Figure 2 and 4, respectively). We find that theta coherence between the generators is not fixed; it rather changes dynamically and is regulated by behaviour. Not surprisingly, when pathway-specific theta oscillations have been taken as the temporal reference instead of the LFP recording, interactions between theta and gamma oscillations were higher (Figure 3). Overall, taking brain oscillations as rhythmic changes in neuronal excitability that can define sequential information packages (Canolty and Knight, 2010; Fries, 2005), the dynamic variation in theta synchrony found in the hippocampus likely reflects multiple thetacoordinated time-frames, with phase differences between oscillations having a large impact on the timing of neuronal firing in the respective layers. Synchronization of theta frameworks will, in turn, coordinate, though not necessarily synchronize (Mizuseki and Buzsaki, 2014), firing sequences in consecutive hippocampal stations. The processing streams thus generated could transmit independent information, e.g. driven by memory retrieval or external environmental cues, or the result of integrating/comparing both information sources, depending on the cognitive needs.

Interactions between the phase of the theta oscillation and the amplitude of the gamma activity have been extensively documented and proposed as an effective mechanism to integrate activity across different spatial and temporal scales (Bragin et al., 1995; Bruns and Eckhorn, 2004; Buzsáki and Draguhn, 2004; Canolty et al., 2006; Canolty and Knight, 2010; 
Colgin, 2015a; Colgin et al., 2009; Engel et al., 2001; Fell and Axmacher, 2011; Lakatos et al., 2008, 2005; Lisman and Idiart, 1995; Lisman and Jensen, 2013; Mormann et al., 2005; Palva et al., 2005; Saleh et al., 2010; Soltesz and Deschênes, 1993; Tort et al., 2009, 2008; Zheng et al., 2016). Our analysis demonstrates that phase-amplitude CFC between theta and gamma oscillations in the hippocampus is a pathway-specific phenomenon (Figure 3). This observation, together with previous and important evidence demonstrating that firing of principal cells in CA3 and EC3 is phase-locked to downstream theta-nested gamma oscillations recorded in the CA1 stratum radiatum $(\mathrm{CA} 1 \gamma \mathrm{S})$ and lacunosum-moleculare $(\mathrm{CA} 1 \gamma \mathrm{M})$, respectively (Colgin et al., 2009; Lasztóczi and Klausberger, 2014; Schomburg et al., 2014), suggest that CFC reflects a local interaction driven by upstream afferences organizing downstream circuits in gamma oscillations. Our CFD analysis further supports this interpretation, since it shows a predominant amplitude-to-phase coupling for the same pathway-specific gamma frequency bands. This does not mean that theta oscillations in the hippocampus are generated by gamma activity, on the contrary, we suggest that gamma activities, reflecting the interplay of inhibitory-excitatory networks (Cardin et al., 2009; Neymotin et al., 2011; Orbán et al., 2006; Rotstein et al., 2005; Stark et al., 2013; Tort et al., 2007), impose phase shifts on the on-going theta oscillations in their corresponding layers. Therefore, local gamma-generating circuits, driven by afferences from their respective upstream layers, might not be activated at a particular theta phase, but rather be coordinating principal cells activity and setting the phase of the local theta oscillation.

This interpretation would also explain phase-phase coupling between CA1 theta and $\mathrm{CA} 1 \gamma \mathrm{S}$ and CA1 $\gamma \mathrm{M}$ (Belluscio et al., 2012), as the consequence of theta phase driven by pathway-specific gamma activity entrained by upstream inputs in CA3 and EC3, respectively. Recent studies, however, highlighted the importance of frequency harmonics and waveform asymmetry when measuring phase-phase coupling (Scheffer-Teixeira and Tort, 2016) and also amplitude-phase CFC (Cole and Voytek, 2017). Waveform asymmetry in oscillatory activity introduce spectral content that cannot be defined solely by sinusoidal components (Amzica and Steriade, 1998) and, therefore, may result in spurious CFC (Aru et al., 2015; Cole and Voytek, 2017; Kramer et al., 2008; Scheffer-Teixeira and Tort, 2016). Several approaches have been developed to overcome these limitations (Cole and Voytek, 2019; Kramer et al., 2008), improving the estimation of the theta phase and minimizing the effect of sharp edges. We applied these methods in our analysis (Suppl. Methods). The specific waveform of an oscillation should not be seen as a problem but as a source of physiological information when 
appropriately considered (Cole and Voytek, 2017). We further introduce CFD as a valuable tool to unveil spurious contributions of harmonics to a measured CFC.

The proposed scenario provides a mechanism to coordinate distributed computations organized in theta waves, by synchronizing theta oscillations in connected regions through theta-gamma CFC. The highly significant positive correlation between CFC strength and theta synchronization found in our study (Figure 4) supports this view. In this way, our results explicitly link theta-gamma CFC (Lisman and Idiart, 1995) and coherence-based communication (Fries, 2005), the first reflecting the mechanism to align higher excitability windows, so that communication channels between specific network nodes are opened. A number of different studies investigating learning and memory processes in the entorhinalhippocampal network have indeed, but separately, reported increases in theta-gamma CFC or synchronization in different frequency bands associated with memory performance (Buzsáki and Draguhn, 2004; Canolty and Knight, 2010; Engel et al., 2001; Lisman and Jensen, 2013). The association of both phenomena in our study has been possible by the separation of pathway-specific LFPs, that otherwise would have render a mixed readout of theta-gamma interactions (Herreras, 2016).

We have suggested a link between CFC and theta synchronization through a gammadriven adjustment of theta phase and frequency (Figure 3 and 5). While dissecting the precise circuit mechanisms supporting the transfer function from gamma activity to theta phase is out of the scope of the present work, several possibilities exist. Computational works have demonstrated that theta-gamma CFC emerges from the interactions between functionally distinct interneuron populations, as the basket and oriens-lacunosum-moleculare (OLM) cells, interconnected in a network of principal cells receiving an external theta rhythm generator, such as the septal input (Neymotin et al., 2011; Orbán et al., 2006; Rotstein et al., 2005; Tort et al., 2007). The on-going theta oscillation is thus modulated by the inhibitory network, whose activity is known to be reflected in the gamma activity in in vivo extracellular recordings (Cardin et al., 2009; Csicsvari et al., 1999; Stark et al., 2013), and is associated to pathwayspecific inputs (Colgin et al., 2009; Lasztóczi and Klausberger, 2014; Schomburg et al., 2014). Subsets of interneurons can phase-lock to different hippocampal rhythms (Klausberger et al., 2003; Klausberger and Somogyi, 2008) and, interestingly, recent findings showed in the CA1 region that some interneurons can specifically phase-lock to $\mathrm{CA} 1 \gamma \mathrm{S}$ and others to $\mathrm{CA} 1 \gamma \mathrm{M}$, supporting the idea that different classes of interneurons drive slow and medium gamma oscillations (Colgin, 2015b; Fernández-Ruiz et al., 2017; Lasztóczi and Klausberger, 2014). 
Thus, an appealing mechanism for gamma-modulation of theta phase would be the control of different interneuron classes by pathway-specific inputs, which would entrain specific gamma networks modulating principal cell excitability and firing in response to on-going theta inputs, advancing or delaying theta phase. Spiking resonance in principal cells may contribute to this mechanism too, since optogenetic activation of basket interneurons (PV-cells) in the hippocampus and neocortex pace pyramidal cell firing in the theta range, by virtue of postinhibitory rebound of $I_{h}$ activity (Stark et al., 2013). In that experiment, theta-band firing of excitatory neurons required rhythmic activation of basket cells, as white noise activation effectively modulated their activity but did not entrained pyramidal theta-band firing (Stark et al., 2013). Feed-forward activation of interneurons from upstream layers or an external rhythmic input (i.e. cholinergic or GABAergic inputs form the septum), are thus required for resonance amplification. Intrinsic cellular properties and network mechanism may thus interact to support gamma-dependent coordination of theta phases across hippocampal layers.

Interactions between slow and fast brain oscillations have been measured in multiple brain regions during perception, attention, learning and memory formation (Buzsáki and Draguhn, 2004; Engel et al., 2001; Lisman and Jensen, 2013). Despite its ubiquitous presence in fundamental cognitive processes, its function is largely unknown. Our results provide a mechanism for binding or segregating distributed computations packed on theta waves and routing the information flow based on theta-gamma cross-frequency coupling. Important questions remain to be answered. How theta synchronization in the hippocampus relates to hippocampal-neocortical interactions (Siapas et al., 2005; Sirota et al., 2008) known to be favoured at theta and beta frequencies (Igarashi et al., 2014; Moreno et al., 2016) and modulated by synaptic plasticity in the hippocampus (Alvarez-Salvado et al., 2014; Canals et al., 2009)? The conditions triggering the coordination between theta-gamma frameworks are not well understood, but given that theta-gamma uncoupling seems to represent an early electrophysiological signature of hippocampal network dysfunction in Alzheimer's disease (Goutagny et al., 2013; Iaccarino et al., 2016; Palop and Mucke, 2009; Verret et al., 2012) as well as for schizophrenia and other psychiatric disorders (Olypher et al., 2006; Phillips and Silverstein, 2003; Uhlhaas and Singer, 2006), further and detailed mechanistic investigations are granted.

\section{Acknowledgments}


We thank Begoña Fernández for excellent technical assistance and Laura Pérez-Cerveza for her input during data pre-processing with ICA. S.C. and D.M. were supported by the Spanish Agency of Research (AEI) under Grant Nos. BFU2015-64380-C2-1-R and -2-R, respectively, co-financed by the European Regional Development Fund (ERDF). S.C. was further supported by AEI and ERDF under Grant No. PGC2018-101055-B-I00, the European Union Horizon 2020 research and innovation programme under Grant Agreement No. 668863 (SyBil-AA) and acknowledges financial support from the Spanish State Research Agency, through the Severo Ochoa Program for Centres of Excellence in R\&D (SEV- 2017-0723). C.R.M. and E.P. acknowledge support from MINECO trough project Nos. TEC2016-80063-C3-3-R and -2-R, respectively. C.R.M. also acknowledges financial support from the Spanish State Research Agency, through the María de Maeztu Program for Units of Excellence in R\&D (MDM-20170711). O.H. was supported by MINECO under Grant No. SAF2016-80100-R. V.J.L. was supported by a predoctoral fellowship La Caixa-Severo Ochoa from Obra Social La Caixa.

\section{Author Contributions}

V.J.L.-M., E.P.-M., E.A.-S., S.C. design the experiments; E.P.-M., E.A.-S. performed experiments; E.P., D.M., O.H., V.J.L.-M., C.R.M. contributed analytical tools; V.J.L.-M. analyzed the data. All authors interpreted the results. V.J.L.-M., C.R.M., S.C. wrote a first version of the manuscript that was reviewed by all authors. S.C. supervised the project.

\section{Declaration of Interests}

The authors declare no competing interests 


\section{References}

Ainge JA, van der Meer MAA, Langston RF, Wood ER. 2007. Exploring the role of context-dependent hippocampal activity in spatial alternation behavior. Hippocampus 17:988-1002. doi:10.1002/hipo.20301

Alonso A, García-Austt E. 1987. Neuronal sources of theta rhythm in the entorhinal cortex of the rat. II. Phase relations between unit discharges and theta field potentials. Exp Brain Res 67:502509.

Alvarez-Salvado E, Pallarés V, Moreno A, Canals S. 2014. Functional MRI of long-term potentiation: imaging network plasticity. Philos Trans $R$ Soc Lond B Biol Sci 369:20130152. doi:10.1098/rstb.2013.0152

Amzica F, Steriade M. 1998. Electrophysiological correlates of sleep delta waves. Electroencephalogr Clin Neurophysiol 107:69-83.

Aru Juhan, Aru Jaan, Priesemann V, Wibral M, Lana L, Pipa G, Singer W, Vicente R. 2015. Untangling cross-frequency coupling in neuroscience. Curr Opin Neurobiol, SI: Brain rhythms and dynamic coordination 31:51-61. doi:10.1016/j.conb.2014.08.002

Barth AM, Domonkos A, Fernandez-Ruiz A, Freund TF, Varga V. 2018. Hippocampal Network Dynamics during Rearing Episodes. Cell Rep 23:1706-1715. doi:10.1016/j.celrep.2018.04.021

Belluscio MA, Mizuseki K, Schmidt R, Kempter R, Buzsáki G. 2012. Cross-frequency phase-phase coupling between $\theta$ and $\gamma$ oscillations in the hippocampus. J Neurosci Off J Soc Neurosci 32:423-435. doi:10.1523/JNEUROSCI.4122-11.2012

Benito N, Fernández-Ruiz A, Makarov VA, Makarova J, Korovaichuk A, Herreras O. 2014. Spatial modules of coherent activity in pathway-specific LFPs in the hippocampus reflect topology and different modes of presynaptic synchronization. Cereb Cortex N Y N 1991 24:1738-1752. doi:10.1093/cercor/bht022

Bland BH, Whishaw IQ. 1976. Generators and topography of hippocampal theta (RSA) in the anaesthetized and freely moving rat. Brain Res 118:259-280.

Bragin A, Jandó G, Nádasdy Z, Hetke J, Wise K, Buzsáki G. 1995. Gamma (40-100 Hz) oscillation in the hippocampus of the behaving rat. J Neurosci Off J Soc Neurosci 15:47-60.

Bruns A, Eckhorn R. 2004. Task-related coupling from high- to low-frequency signals among visual cortical areas in human subdural recordings. Int J Psychophysiol Off J Int Organ Psychophysiol 51:97-116.

Buzsáki G. 2002. Theta oscillations in the hippocampus. Neuron 33:325-340.

Buzsáki G, Draguhn A. 2004. Neuronal oscillations in cortical networks. Science 304:1926-1929. doi:10.1126/science.1099745

Buzsáki G, Moser El. 2013. Memory, navigation and theta rhythm in the hippocampal-entorhinal system. Nat Neurosci 16:130-138. doi:10.1038/nn.3304

Canals S, Beyerlein M, Merkle H, Logothetis NK. 2009. Functional MRI evidence for LTP-induced neural network reorganization. Curr Biol CB 19:398-403. doi:10.1016/j.cub.2009.01.037

Canolty RT, Edwards E, Dalal SS, Soltani M, Nagarajan SS, Kirsch HE, Berger MS, Barbaro NM, Knight RT. 2006. High Gamma Power Is Phase-Locked to Theta Oscillations in Human Neocortex. Science 313:1626-1628. doi:10.1126/science.1128115

Canolty RT, Knight RT. 2010. The functional role of cross-frequency coupling. Trends Cogn Sci 14:506515. doi:10.1016/j.tics.2010.09.001

Cardin JA, Carlén M, Meletis K, Knoblich U, Zhang F, Deisseroth K, Tsai L-H, Moore Cl. 2009. Driving fast-spiking cells induces gamma rhythm and controls sensory responses. Nature 459:663667. doi:10.1038/nature08002

Charpak S, Paré D, Llinás R. 1995. The entorhinal cortex entrains fast CA1 hippocampal oscillations in the anaesthetized guinea-pig: role of the monosynaptic component of the perforant path. Eur J Neurosci 7:1548-1557.

Cole S, Voytek B. 2019. Cycle-by-cycle analysis of neural oscillations. I Neurophysiol. doi:10.1152/jn.00273.2019 
bioRxiv preprint doi: https://doi org/10.1101/418434; this version posted March 26, 2020. The copyright holder for this preprint (which was not certified by peer review) is the author/funder, who has granted bioRxiv a license to display the preprint in perpetuity. It is made available under aCC-BY 4.0 International license.

Cole SR, Voytek B. 2017. Brain Oscillations and the Importance of Waveform Shape. Trends Cogn Sci 21:137-149. doi:10.1016/j.tics.2016.12.008

Colgin LL. 2016. Rhythms of the hippocampal network. Nat Rev Neurosci 17:239-249. doi:10.1038/nrn.2016.21

Colgin LL. 2015a. Theta-gamma coupling in the entorhinal-hippocampal system. Curr Opin Neurobiol, SI: Brain rhythms and dynamic coordination 31:45-50. doi:10.1016/j.conb.2014.08.001

Colgin LL. 2015b. Theta-gamma coupling in the entorhinal-hippocampal system. Curr Opin Neurobiol, SI: Brain rhythms and dynamic coordination 31:45-50. doi:10.1016/j.conb.2014.08.001

Colgin LL. 2013. Mechanisms and functions of theta rhythms. Annu Rev Neurosci 36:295-312. doi:10.1146/annurev-neuro-062012-170330

Colgin LL, Denninger T, Fyhn M, Hafting T, Bonnevie T, Jensen O, Moser M-B, Moser El. 2009. Frequency of gamma oscillations routes flow of information in the hippocampus. Nature 462:353-357. doi:10.1038/nature08573

Csicsvari J, Hirase H, Czurkó A, Mamiya A, Buzsáki G. 1999. Oscillatory coupling of hippocampal pyramidal cells and interneurons in the behaving Rat. J Neurosci Off J Soc Neurosci 19:274287.

DeCoteau WE, Thorn C, Gibson DJ, Courtemanche R, Mitra P, Kubota Y, Graybiel AM. 2007. Learningrelated coordination of striatal and hippocampal theta rhythms during acquisition of a procedural maze task. Proc Natl Acad Sci 104:5644-5649. doi:10.1073/pnas.0700818104

Douchamps V, Jeewajee A, Blundell P, Burgess N, Lever C. 2013. Evidence for encoding versus retrieval scheduling in the hippocampus by theta phase and acetylcholine. J Neurosci Off J Soc Neurosci 33:8689-8704. doi:10.1523/JNEUROSCI.4483-12.2013

Dudai Y, Morris RGM. 2013. Memorable trends. Neuron 80:742-750. doi:10.1016/j.neuron.2013.09.039

Engel AK, Fries P, Singer W. 2001. Dynamic predictions: oscillations and synchrony in top-down processing. Nat Rev Neurosci 2:704-716. doi:10.1038/35094565

Fell J, Axmacher N. 2011. The role of phase synchronization in memory processes. Nat Rev Neurosci 12:105-118. doi:10.1038/nrn2979

Fernández-Ruiz A, Herreras O. 2013. Identifying the synaptic origin of ongoing neuronal oscillations through spatial discrimination of electric fields. Front Comput Neurosci 7:5. doi:10.3389/fncom.2013.00005

Fernández-Ruiz A, Makarov VA, Benito N, Herreras O. 2012. Schaffer-specific local field potentials reflect discrete excitatory events at gamma frequency that may fire postsynaptic hippocampal CA1 units. J Neurosci Off J Soc Neurosci 32:5165-5176. doi:10.1523/JNEUROSCI.4499-11.2012

Fernández-Ruiz A, Oliva A, Nagy GA, Maurer AP, Berényi A, Buzsáki G. 2017. Entorhinal-CA3 Dual-Input Control of Spike Timing in the Hippocampus by Theta-Gamma Coupling. Neuron 93:12131226.e5. doi:10.1016/j.neuron.2017.02.017

Fries P. 2015. Rhythms for Cognition: Communication through Coherence. Neuron 88:220-235. doi:10.1016/j.neuron.2015.09.034

Fries P. 2005. A mechanism for cognitive dynamics: neuronal communication through neuronal coherence. Trends Cogn Sci 9:474-480. doi:10.1016/j.tics.2005.08.011

Goutagny R, Gu N, Cavanagh C, Jackson J, Chabot J-G, Quirion R, Krantic S, Williams S. 2013. Alterations in hippocampal network oscillations and theta-gamma coupling arise before $A \beta$ overproduction in a mouse model of Alzheimer's disease. Eur J Neurosci 37:1896-1902. doi:10.1111/ejn.12233

Granger CWJ. 1969. Investigating Causal Relations by Econometric Models and Cross-spectral Methods. Econometrica 37:424-438. doi:10.2307/1912791

Green KF, Rawlins JN. 1979. Hippocampal theta in rats under urethane: generators and phase relations. Electroencephalogr Clin Neurophysiol 47:420-429.

H. Vanderwolf C, Bland B, Whishaw I. 1973. Diencephalic, Hippocampal, and Neocortical Mechanisms in Voluntary Movement. Efferent Organ Integr Behav. doi:10.1016/B978-0-12-4769502.50014-2 
Hasselmo ME, Bodelón C, Wyble BP. 2002. A proposed function for hippocampal theta rhythm: separate phases of encoding and retrieval enhance reversal of prior learning. Neural Comput 14:793-817. doi:10.1162/089976602317318965

Herreras O. 2016. Local Field Potentials: Myths and Misunderstandings. Front Neural Circuits 10:101. doi:10.3389/fncir.2016.00101

laccarino HF, Singer AC, Martorell AJ, Rudenko A, Gao F, Gillingham TZ, Mathys H, Seo J, Kritskiy O, Abdurrob F, Adaikkan C, Canter RG, Rueda R, Brown EN, Boyden ES, Tsai L-H. 2016. Gamma frequency entrainment attenuates amyloid load and modifies microglia. Nature 540:230-235. doi:10.1038/nature20587

Igarashi KM, Lu L, Colgin LL, Moser M-B, Moser El. 2014. Coordination of entorhinal-hippocampal ensemble activity during associative learning. Nature 510:143-147. doi:10.1038/nature13162

Jiang H, Bahramisharif A, van Gerven MAJ, Jensen O. 2015. Measuring directionality between neuronal oscillations of different frequencies. Neurolmage 118:359-367. doi:10.1016/j.neuroimage.2015.05.044

Klausberger T, Magill PJ, Márton LF, Roberts JDB, Cobden PM, Buzsáki G, Somogyi P. 2003. Brain-stateand cell-type-specific firing of hippocampal interneurons in vivo. Nature 421:844-848. doi:10.1038/nature01374

Klausberger T, Somogyi P. 2008. Neuronal diversity and temporal dynamics: the unity of hippocampal circuit operations. Science 321:53-57. doi:10.1126/science.1149381

Kocsis B, Bragin A, Buzsáki G. 1999. Interdependence of Multiple Theta Generators in the Hippocampus: a Partial Coherence Analysis. J Neurosci 19:6200-6212.

Kramer MA, Tort ABL, Kopell NJ. 2008. Sharp edge artifacts and spurious coupling in EEG frequency comodulation measures. $J$ Neurosci Methods 170:352-357. doi:10.1016/j.jneumeth.2008.01.020

Kramis R, Vanderwolf $\mathrm{CH}$, Bland $\mathrm{BH}$. 1975. Two types of hippocampal rhythmical slow activity in both the rabbit and the rat: relations to behavior and effects of atropine, diethyl ether, urethane, and pentobarbital. Exp Neurol 49:58-85.

Lakatos P, Karmos G, Mehta AD, Ulbert I, Schroeder CE. 2008. Entrainment of neuronal oscillations as a mechanism of attentional selection. Science 320:110-113. doi:10.1126/science.1154735

Lakatos P, Shah AS, Knuth KH, Ulbert I, Karmos G, Schroeder CE. 2005. An oscillatory hierarchy controlling neuronal excitability and stimulus processing in the auditory cortex. J Neurophysiol 94:1904-1911. doi:10.1152/jn.00263.2005

Lasztóczi B, Klausberger T. 2016. Hippocampal Place Cells Couple to Three Different Gamma Oscillations during Place Field Traversal. Neuron 91:34-40. doi:10.1016/j.neuron.2016.05.036

Lasztóczi B, Klausberger T. 2014. Layer-specific GABAergic control of distinct gamma oscillations in the CA1 hippocampus. Neuron 81:1126-1139. doi:10.1016/j.neuron.2014.01.021

Lever C, Burton S, O'Keefe J. 2006. Rearing on hind legs, environmental novelty, and the hippocampal formation. Rev Neurosci 17:111-133. doi:10.1515/revneuro.2006.17.1-2.111

Lisman JE, Idiart MA. 1995. Storage of 7 +/- 2 short-term memories in oscillatory subcycles. Science 267:1512-1515.

Lisman JE, Jensen 0 . 2013. The $\theta-\gamma$ neural code. Neuron 77:1002-1016. doi:10.1016/j.neuron.2013.03.007

Makarov VA, Makarova J, Herreras O. 2010. Disentanglement of local field potential sources by independent component analysis. J Comput Neurosci 29:445-457. doi:10.1007/s10827-0090206-y

Makarova J, Ibarz JM, Makarov VA, Benito N, Herreras O. 2011. Parallel readout of pathway-specific inputs to laminated brain structures. Front Syst Neurosci 5:77. doi:10.3389/fnsys.2011.00077

Martín-Vázquez G, Makarova J, Makarov VA, Herreras O. 2013. Determining the true polarity and amplitude of synaptic currents underlying gamma oscillations of local field potentials. PloS One 8:e75499. doi:10.1371/journal.pone.0075499 
bioRxiv preprint doi: https://doi org/10.1101/418434; this version posted March 26, 2020. The copyright holder for this preprint (which was not certified by peer review) is the author/funder, who has granted bioRxiv a license to display the preprint in perpetuity. It is made available under aCC-BY 4.0 International license.

McNaughton BL, Barnes CA, O'Keefe J. 1983. The contributions of position, direction, and velocity to single unit activity in the hippocampus of freely-moving rats. Exp Brain Res 52:41-49. doi:10.1007/bf00237147

Mizuseki K, Buzsaki G. 2014. Theta oscillations decrease spike synchrony in the hippocampus and entorhinal cortex. Philos Trans $R$ Soc Lond B Biol Sci 369:20120530. doi:10.1098/rstb.2012.0530

Mizuseki K, Sirota A, Pastalkova E, Buzsáki G. 2009. Theta oscillations provide temporal windows for local circuit computation in the entorhinal-hippocampal loop. Neuron 64:267-280. doi:10.1016/j.neuron.2009.08.037

Montgomery SM, Betancur MI, Buzsáki G. 2009. Behavior-dependent coordination of multiple theta dipoles in the hippocampus. J Neurosci Off J Soc Neurosci 29:1381-1394. doi:10.1523/JNEUROSCI.4339-08.2009

Montgomery SM, Buzsáki G. 2007. Gamma oscillations dynamically couple hippocampal CA3 and CA1 regions during memory task performance. Proc Natl Acad Sci U S A 104:14495-14500. doi:10.1073/pnas.0701826104

Moreno A, Morris RGM, Canals S. 2016. Frequency-Dependent Gating of Hippocampal-Neocortical Interactions. Cereb Cortex N Y N 1991 26:2105-2114. doi:10.1093/cercor/bhv033

Mormann F, Fell J, Axmacher N, Weber B, Lehnertz K, Elger CE, Fernández G. 2005. Phase/amplitude reset and theta-gamma interaction in the human medial temporal lobe during a continuous word recognition memory task. Hippocampus 15:890-900. doi:10.1002/hipo.20117

Neymotin SA, Lazarewicz MT, Sherif M, Contreras D, Finkel LH, Lytton WW. 2011. Ketamine disrupts $\theta$ modulation of $\gamma$ in a computer model of hippocampus. J Neurosci Off J Soc Neurosci 31:11733-11743. doi:10.1523/JNEUROSCI.0501-11.2011

Nolte G, Ziehe A, Krämer N, Popescu F, Müller K-R. 2010. Comparison of Granger Causality and Phase Slope IndexCausality: Objectives and Assessment. Presented at the Causality: Objectives and Assessment. pp. 267-276.

O'Keefe J, Nadel L. 1978. The hippocampus as a cognitive map. Oxford; New York: Clarendon Press ; Oxford University Press.

Olypher AV, Klement D, Fenton AA. 2006. Cognitive disorganization in hippocampus: a physiological model of the disorganization in psychosis. I Neurosci Off J Soc Neurosci 26:158-168. doi:10.1523/JNEUROSCI.2064-05.2006

Orbán G, Kiss T, Erdi P. 2006. Intrinsic and synaptic mechanisms determining the timing of neuron population activity during hippocampal theta oscillation. J Neurophysiol 96:2889-2904. doi:10.1152/jn.01233.2005

Palop JJ, Mucke L. 2009. Epilepsy and cognitive impairments in Alzheimer disease. Arch Neurol 66:435440. doi:10.1001/archneurol.2009.15

Palva JM, Palva S, Kaila K. 2005. Phase synchrony among neuronal oscillations in the human cortex. J Neurosci Off J Soc Neurosci 25:3962-3972. doi:10.1523/JNEUROSCI.4250-04.2005

Pastalkova E, Itskov V, Amarasingham A, Buzsáki G. 2008. Internally generated cell assembly sequences in the rat hippocampus. Science 321:1322-1327. doi:10.1126/science.1159775

Phillips WA, Silverstein SM. 2003. Convergence of biological and psychological perspectives on cognitive coordination in schizophrenia. Behav Brain Sci 26:65-82; discussion 82-137.

Rotstein HG, Pervouchine DD, Acker CD, Gillies MJ, White JA, Buhl EH, Whittington MA, Kopell N. 2005. Slow and fast inhibition and an $\mathrm{H}$-current interact to create a theta rhythm in a model of CA1 interneuron network. J Neurophysiol 94:1509-1518. doi:10.1152/jn.00957.2004

Saleh M, Reimer J, Penn R, Ojakangas CL, Hatsopoulos NG. 2010. Fast and slow oscillations in human primary motor cortex predict oncoming behaviorally relevant cues. Neuron 65:461-471. doi:10.1016/j.neuron.2010.02.001

Scheffer-Teixeira R, Tort AB. 2016. On cross-frequency phase-phase coupling between theta and gamma oscillations in the hippocampus. elife 5. doi:10.7554/elife.20515 
Schomburg EW, Fernández-Ruiz A, Mizuseki K, Berényi A, Anastassiou CA, Koch C, Buzsáki G. 2014. Theta Phase Segregation of Input-Specific Gamma Patterns in Entorhinal-Hippocampal Networks. Neuron 84:470-485. doi:10.1016/j.neuron.2014.08.051

Sheremet A, Burke SN, Maurer AP. 2016. Movement Enhances the Nonlinearity of Hippocampal Theta. J Neurosci Off J Soc Neurosci 36:4218-4230. doi:10.1523/JNEUROSCI.3564-15.2016

Sheremet A, Kennedy JP, Qin Y, Zhou Y, Lovett SD, Burke SN, Maurer AP. 2019. Theta-gamma cascades and running speed. J Neurophysiol 121:444-458. doi:10.1152/jn.00636.2018

Siapas AG, Lubenov EV, Wilson MA. 2005. Prefrontal phase locking to hippocampal theta oscillations. Neuron 46:141-151. doi:10.1016/j.neuron.2005.02.028

Siegle JH, Wilson MA. 2014. Enhancement of encoding and retrieval functions through theta phasespecific manipulation of hippocampus. eLife 3:e03061. doi:10.7554/eLife.03061

Sirota A, Montgomery S, Fujisawa S, Isomura Y, Zugaro M, Buzsáki G. 2008. Entrainment of neocortical neurons and gamma oscillations by the hippocampal theta rhythm. Neuron 60:683-697. doi:10.1016/j.neuron.2008.09.014

Soltesz I, Deschênes M. 1993. Low- and high-frequency membrane potential oscillations during theta activity in CA1 and CA3 pyramidal neurons of the rat hippocampus under ketamine-xylazine anesthesia. J Neurophysiol 70:97-116. doi:10.1152/jn.1993.70.1.97

Stark E, Eichler R, Roux L, Fujisawa S, Rotstein HG, Buzsáki G. 2013. Inhibition-induced theta resonance in cortical circuits. Neuron 80:1263-1276. doi:10.1016/j.neuron.2013.09.033

Tort ABL, Komorowski RW, Manns JR, Kopell NJ, Eichenbaum H. 2009. Theta-gamma coupling increases during the learning of item-context associations. Proc Natl Acad Sci 106:2094220947. doi:10.1073/pnas.0911331106

Tort ABL, Kramer MA, Thorn C, Gibson DJ, Kubota Y, Graybiel AM, Kopell NJ. 2008. Dynamic crossfrequency couplings of local field potential oscillations in rat striatum and hippocampus during performance of a T-maze task. Proc Natl Acad Sci U S A 105:20517-20522. doi:10.1073/pnas.0810524105

Tort ABL, Rotstein HG, Dugladze T, Gloveli T, Kopell NJ. 2007. On the formation of gamma-coherent cell assemblies by oriens lacunosum-moleculare interneurons in the hippocampus. Proc Natl Acad Sci U S A 104:13490-13495. doi:10.1073/pnas.0705708104

Uhlhaas PJ, Singer W. 2006. Neural synchrony in brain disorders: relevance for cognitive dysfunctions and pathophysiology. Neuron 52:155-168. doi:10.1016/j.neuron.2006.09.020

Vanderwolf $\mathrm{CH}$. 1969. Hippocampal electrical activity and voluntary movement in the rat. Electroencephalogr Clin Neurophysiol 26:407-418.

Verret L, Mann EO, Hang GB, Barth AMI, Cobos I, Ho K, Devidze N, Masliah E, Kreitzer AC, Mody I, Mucke L, Palop JJ. 2012. Inhibitory interneuron deficit links altered network activity and cognitive dysfunction in Alzheimer model. Cell 149:708-721. doi:10.1016/j.cell.2012.02.046

Wang S-H, Morris RGM. 2010. Hippocampal-neocortical interactions in memory formation, consolidation, and reconsolidation. Annu Rev Psychol 61:49-79, C1-4. doi:10.1146/annurev.psych.093008.100523

Wang Y, Romani S, Lustig B, Leonardo A, Pastalkova E. 2015. Theta sequences are essential for internally generated hippocampal firing fields. Nat Neurosci 18:282-288. doi:10.1038/nn.3904

Wells CE, Amos DP, Jeewajee A, Douchamps V, Rodgers J, O'Keefe J, Burgess N, Lever C. 2013. Novelty and anxiolytic drugs dissociate two components of hippocampal theta in behaving rats. $J$ Neurosci Off J Soc Neurosci 33:8650-8667. doi:10.1523/JNEUROSCI.5040-12.2013

Winson J. 1974. Patterns of hippocampal theta rhythm in the freely moving rat. Electroencephalogr Clin Neurophysiol 36:291-301.

Wood ER, Dudchenko PA, Robitsek RJ, Eichenbaum H. 2000. Hippocampal neurons encode information about different types of memory episodes occurring in the same location. Neuron 27:623-633.

Zheng C, Bieri KW, Hwaun E, Colgin LL. 2016. Fast Gamma Rhythms in the Hippocampus Promote Encoding of Novel Object-Place Pairings. eNeuro 3. doi:10.1523/ENEURO.0001-16.2016 
bioRxiv preprint doi: https://doi.org/10.1101/418434; this version posted March 26, 2020. The copyright holder for this preprint (which was not certified by peer review) is the author/funder, who has granted bioRxiv a license to display the preprint in perpetuity. It is made available under aCC-BY 4.0 International license.

Zhou Y, Sheremet A, Qin Y, Kennedy JP, DiCola NM, Burke SN, Maurer AP. 2019. Methodological Considerations on the Use of Different Spectral Decomposition Algorithms to Study Hippocampal Rhythms. eNeuro 6. doi:10.1523/ENEURO.0142-19.2019 


\section{SUPPLEMENTARY INFORMATION (SI)}

Different theta frameworks coexist in the hippocampus and are coordinated during memory-guided exploration and novelty detection.

Víctor J. Lopez-Madrona ${ }^{1}$, Elena Pérez-Montoyo ${ }^{1}$, Efrén Álvarez-Salvado ${ }^{1}$, David Moratal ${ }^{2}$, Oscar Herreras $^{3}$, Ernesto Pereda ${ }^{4,5}$, Claudio R. Mirasso ${ }^{6}$, Santiago Canals ${ }^{1}$

1 Instituto de Neurociencias, Consejo Superior de Investigaciones Científicas, Universidad Miguel Hernández, San Juan de Alicante 03550, Spain

2 Centro de Biomateriales e Ingeniería Tisular, Universitat Politècnica de València, Valencia 46022, Spain

3 Instituto Cajal, Consejo Superior de Investigaciones Científicas, Madrid 28002, Spain

4 Departamento de Ingeniería Industrial \& IUNE, Escuela Superior de Ingeniería y Tecnología, Universidad de La Laguna, La Laguna, Tenerife 38205, Spain

5 Laboratory of Cognitive and Computational Neuroscience, Center for Biomedical Technology, Madrid, Spain

6 Instituto de Física Interdisciplinar y Sistemas Complejos, IFISC (CSIC-UIB), Campus Universitat de les Illes Balears, Palma de Mallorca 07122, Spain

Corresponding Author: Santiago Canals (scanals@umh.es) 


\section{Methods}

All animal experiments were approved by the Animal Care and Use Committee of the Instituto de Neurociencias de Alicante, Alicante, Spain, and comply with the Spanish (law 32/2007) and European regulations (EU directive 86/609, EU decree 2001-486, and EU recommendation 2007/526/EC).

\section{Animals and Surgery}

Five male Long-Evans rats, with a weigh of 250-300 g. were trained in different behavioral tasks, with a multichannel electrode recording the electrophysiological activity in the hippocampus. All of them were implanted with a 32 channels silicon probe (Neuronexus Technologies, Michigan, USA) connected in turn to a jumper consisting of two corresponding connectors joined by $5 \mathrm{~cm}$ of flexible cable. $\mathrm{An} \mathrm{Ag} / \mathrm{AgCl}$ wire (World Precision Instruments, Florida, USA) electrode was placed in contact with the skin on the sides of the surgery area, and used as ground. The data was acquired at $5 \mathrm{kHz}$, with an analog high-pass filter at $0.5 \mathrm{~Hz}$. After digitalization, we initially low-pass filtered them at $300 \mathrm{~Hz}$, removed the net noise with Notch filters at $50 \mathrm{~Hz}$ and $100 \mathrm{~Hz}$ and down-sampled the signals at $2.5 \mathrm{kHz}$. We adjusted the final position of both electrodes using as a reference the typical evoked potentials at the dentate gyrus (Andersen et al., 1966), so that a maximal population spike in the dentate gyrus was recorded.

After the surgery, the rats were left for at least 10 days until they recovered completely. During the first 72 hours, they were injected subcutaneously with analgesic twice per day (Buprenorphine, dose 2-5 $\mu \mathrm{g} / \mathrm{kg}$, RB Pharmaceutical Ltd., Berkshire, UK). During 1 week, they had as well antibiotic dissolved in the water (Enrofloxacin, dose $10 \mathrm{mg} / \mathrm{kg}$, Syva, León, Spain). The behavioral tasks were not started until the animals showed no signs of discomfort with the manipulation of the implants.

\section{$\underline{\text { Data acquisition }}$}

All subjects were trained before the surgery following the next protocol. The first three days consisted on a habituation process with two 10-minutes sessions per day in an open field, with freedom of movement. The environment was a methacrylate sandbox of $50 \times 50 \mathrm{~cm}$, opened at the top and with three visual cues in three of the walls. After that, they carried out two new sessions per day for 8 days, first repeating the habituation and then performing a modified Tmaze task, that has been described previously (Wood et al., 2000). It consisted in several tracks in 8-like shape (132/102/80 $\mathrm{cm}$ long/wide/high with track wide $8 \mathrm{~cm}$, Figure 6e). The starting 
point was located at the beginning of the central rail (Figure 6e, start) and the rat was forced to run across that arm (Figure 6e, centre), blocking other pathways with black panels. At the end of the track, it must choose one of the two directions of the T-junction and a small drop of water was delivered at the corner (Figure 6e, reward) in successfully trials. Each repetition is considered successful if the rat chooses the opposite direction with respect to the previous trial, finding always a reward at the corner after the T-junction. Then, another panel located after the water prevented the rat from retracing its route, forcing it to go to the starting point across the corresponding side arm (Figure 6e, side), for a new trial. Each session had a duration of 20 minutes with around 30 trials, and all the subjects reached a performance greater than $80 \%$ in the last session. Only correct trials were considered for further analysis.

After the surgery and recovery, we repeated the same protocol for 8 days. For further electrophysiological analysis, we considered only those sessions were the subjects kept a high level of performance $(80 \%)$ without any interference. There were in total between 2 and 5 sessions of 4 subjects. During the $9^{\text {th }}$ day, we carried out a "novelty" test. The rats were exposed to a novelty by introducing them in a "novelty chamber" located inside the familiar open field; such chamber was a transparent methacrylate box with a square base $35 \mathrm{~cm}$ wide, and $40 \mathrm{~cm}$ high, opened at the top, with sand paper on the floor to provide a noticeable tactile stimulus. After this time, the novelty chamber was removed, and the animals were left another 10 minutes in the open field, considering this session as the control condition for the analysis.

Except for the results in figure 6, all the analyses were carried out during the control session (last session), with freedom of movement in a well-known environment.

\section{Optogenetic experiments}

Four male Long-Evans transgenic rats, expressing Cre recombinase under the rat parvalbumin promoter (LE-TG[Pvalb-iCre]2Ottc, NIDA, USA), were bred in our facilities, housed in pairs with food and water available ad libitum and maintained on a 12/12h light-dark cycle.

\section{Virus injection and surgeries}

For the surgery, rats were anesthetized with isoflurane (4.5\% induction, $1-2 \%$ maintenance, in $\left.0.8 \mathrm{l} / \mathrm{min} \mathrm{O}_{2}\right)$ and locally anesthetized by subcutaneous injection of bupivacaine $(0.2 \mathrm{ml})$. All rats weighted 300-330 gr at the time of the first surgery. The Cre-dependent viral vector AAV1EF1a-DIO-hChR2(H134R)-eYFP-WPRE-hGH (Penn Vector Core) was bilaterally injected in dorsal CA3 (AP $-3.5 \mathrm{~mm}, \mathrm{LM} \pm 3.6 \mathrm{~mm}$ from bregma and DV $-2.8 \mathrm{~mm}$ from the brain surface) 
using a Hamilton syringe attached to an infusion pump ( $1 \mu \mathrm{l}$ per hemisphere at $1 \mu \mathrm{l} / \mathrm{min})$. Thus, ChR2 is specifically expressed in PV+ cells (PV-ChR2).

Two weeks after the virus injection, rats underwent a second surgery for two fiber-optic cannulas and one recording electrode implantation. First, five screws were attached to the skull to strengthen the fixation of the implant. As in the previous group of rats, a 32 channels silicon probe connected to a jumper (Neuronexus Technologies, Michigan, USA) was placed in the left hippocampus covering dorsal CA1 and DG. Reference wires were attached to one of the screws. The coordinates for the electrode implantation were AP $-3.5 \mathrm{~mm}, \mathrm{LM} \pm 2.5 \mathrm{~mm}$ from bregma and DV $-3.0 \mathrm{~mm}$ from the brain surface, although its final position was adjusted based on the electrophysiological potentials evoked by stimulating the perforant pathway. Then, a fiber-optic cannula (200 $\mu$ m diameter, 0.66 NA, 10 mm length; Doric Lenses, Quebec, Canada) was placed in the dorsal CA3 of both hemispheres with an angle of $20^{\circ}$ in the coronal plane at the coordinates AP $-3.5 \mathrm{~mm}, \mathrm{LM} \pm 5.2 \mathrm{~mm}$ from bregma and $\mathrm{DV}-3.2 \mathrm{~mm}$ from the brain surface (Figure 2a). Once the electrode and the two fibers were positioned, the stimulation electrode was removed and several layers of dental cement (SuperBond or Palacos) were applied to ensure enough fixation of all the components. The post-operative care was the same as in the previous group of rats (see above). One of the subjects was excluded from the experiment after the surgery and prior to any analysis due to the reduced quality of its electrophysiological recordings.

\section{Optogenetic manipulation in behaving animals and data acquisition}

All behavioral procedures were conducted during the dark cycle. After the complete recovery following the implantation surgery and before starting the experiments, we handled the animals during 5 days in order to habituate them to the experimenter as well as to the manipulation of the implant (connection of the headstage and the fiber-optic patch cords).

Rats performed an 8-minutes daily session during 5 consecutive days in an already known open field (50x50x40cm box of black methacrylate) with bedding covering the floor. Animals were allowed to freely explore the arena in each session while receiving ON/OFF periods of light stimulation. The light for excitation of ChR2 was delivered at $50 \mathrm{~mW} / \mathrm{mm}^{2}$ by a blue LED source (Prizmatix, Canada) at a wavelength of $460 \mathrm{~nm}$. Stimulation protocol consisted on 5 seconds $40-\mathrm{Hz}$ trains with $1-\mathrm{ms}$ light pulses each 30 seconds during the entire session.

For the bilateral stimulation, we used a branching fiber-optic patch cord (500 $\mu \mathrm{m}$ diameter, $0.63 \mathrm{NA}$; Doric Lenses) connected to a rotatory joint (Prizmatix) which in turn connects to the 
LED source by a fiber-optic patch cord ( $1 \mathrm{~mm}$ diameter, $0.63 \mathrm{NA}$; Doric Lenses). The power density of the delivered light was measured prior to each session using a powermeter (Thorlabs) to ensure the same power density in all sessions $\left(50 \mathrm{~mW} / \mathrm{mm}^{2}\right)$.

Light pulses were triggered by a stimulus generator (STG2004, Multichannel Systems, Reutlingen, Germany) controlled by MC_Stimulus software (Multichannel Systems). Electrophysiological data was recorded at $5 \mathrm{kHz}$ sampling rate with an open-source acquisition system (Open Ephys) and synchronized with light stimulation and video recording by using an I/O board (Open Ephys).

\section{Inmunohystochemical analysis}

After the performance of the experiments, the rats were perfused intracardially with PFA $4 \%$. Brains were kept in post-fixation for $3 \mathrm{~h}$ at $\mathrm{RT}$ and then stored in $\mathrm{PBS}$ at $4^{\circ} \mathrm{Co} / \mathrm{n}$. Then, brains were cut in $50 \mu \mathrm{m}$-slices to corroborate the viral infection as well as the correct position of the recording electrode and the fiber-optic cannulas. Slices were incubated with monoclonal PV antibody developed in mouse (1:2000, Swant, Switzerland) and afterwards with an anti-mouse secondary antibody developed in goat (1:500, Alexa Fluor 594 dye, Life Technologies, USA). After completion of histological treatments, brain sections were imaged using a fluorescence microscope (DM4000B, Leica) coupled to a Neurolucida software (MicroBrightField, Inc.) and images were processed with Image $\mathbf{J}$ software.

\section{$\underline{\text { Video recording and tracking }}$}

The animals were monitored during all the tasks, and their behavior was recorded using a standard camera located at the top of the room. Using those videos, the location of the subjects was tracked with a custom-made matlab script, taking their centroid as the refence point. The synchronization of the video and the electrophysiological recordings was made triggering a red LED and matching the temporal mark that it left in the recordings with the first frame with light.

\section{Current source density analysis of LFPs}

The first approach to achieve the information of the sources contributing to the LFPs was the use of current source density (Freeman and Nicholson, 1975; Herreras, 1990; Holsheimer, 1987; Mitzdorf, 1985) (CSD) analysis. It measures the transmembrane currents, providing a spatiotemporal distribution of the local sinks and sources (inward and outward currents, 
respectively). Contrary to the LFPs, these currents represent spatially localized phenomena, increasing the spatial resolution.

The membrane currents can be achieved following the Laplace equation and using the measured field potentials and the conductivity of the medium. As the hippocampus is a layered structure, the one-dimensional approach in the direction parallel to the recording electrode was used:

$$
C S D_{m}(t)=-\frac{\sigma}{h^{2}}\left(u_{m-1}(t)-2 u_{m}(t)+u_{m+1}(t)\right)
$$

where $u_{m}(t)$ is the LFP recorded at the m-th site, $h$ is the distance between channels and $\sigma$ is the conductivity of the extracellular space.

We assumed the whole structure as an isotropic and homogeneous medium. Though hippocampal strata present different resistivities, they do not affect much to the temporal dynamics of specific locations (Herreras, 1990; Holsheimer, 1987; López-Aguado et al., 2001). Therefore, the distance and conductivity are constants, and they merely act as a scale factor. In this work, the distance between contacts was $h=100 \mu \mathrm{m}$ and we assumed a constant conductivity $\sigma=350 \Omega^{-1} \mathrm{~cm}^{-1}$ (López-Aguado et al., 2001).

Though the CSD presents higher spatial resolution than the LFPs, it does not discriminate contributions from different pathways. Multiple membrane currents with different origins may overlap spatiotemporally, and local currents are also affected by the activity in nearby domains (Herreras, 2016; Korovaichuk et al., 2010; Martín-Vázquez et al., 2013). To overcome these limitations, we applied an independent component analysis (ICA).

\section{Independent Component Analysis of LFPs}

The LFP represent the mixed activity of multiple local and remote populations. Methods as the CSD allows to eliminate the propagated field potentials and extract the local transmembrane currents (see above). Nevertheless, different pathways are contributing to these currents, and their activities may overlap spatiotemporally. To disentangle the specific sources that generate the LFPs, we applied an ICA.

The effectiveness of this approach has been well studied and established in the hippocampus (Fernández-Ruiz et al., 2012b; Herreras et al., 2015; Korovaichuk et al., 2010; Makarov et al., 2010; Makarova et al., 2011; Schomburg et al., 2014). It aims to solve the problem of separating $\mathrm{N}$ statistically independent sources that have been mixed in M output channels. To 
do that, it performs a blind separation of patterns, because the different distributions of the sources are unknown. Moreover, it assumes spatial immobility of the sources or, in other terms, a fixed location of the axon terminals. The contribution of their synaptic currents to the LFP conforms the different independent components (ICs) or generators to unravel.

Each recorded time-series $u_{m}(t)$ is modeled as the sum of $\mathrm{N}$ neuronal sources multiplied by a constant factor:

$$
u_{m}(t)=\sum_{n=1}^{N} V_{m n} s_{n}(t), \quad m=1,2, \ldots, M
$$

where $V_{m n}$ is the mixing matrix with the voltage loadings of N LFP generators on M electrodes and $s_{n}(t)$ is the time-series associated to the n-th LFP generator.

As the number of ICs with significant variance is usually low (4-7 out of 32) (Benito et al., 2014; Korovaichuk et al., 2010), we applied a dimension reduction of the loading matrix by prior use of principal component analysis, keeping $99 \%$ of the original LFP variance. For each structure and electrographic state, the number of optimal components is determined by stepwise increase of the number of principal components until the new ICs are only noise (Makarov et al., 2010). Since noisy components contribute negligible variance (in absence of artefacts in the signal) we always choose this number plus two. There are several algorithms to compute the mixing matrix that transforms LFP data into ICs, nevertheless, all of them share a common theory framework. In this work, we have used the information-maximization approach RUNICA (Bell and Sejnowski, 1995), implemented in the matlab toolbox "ICAofLFPs" (http://www.mat.ucm.es/ vmakarov/downloads.php). For comparison porpoises, the kernel density ICA algorithm KDICA (Chen, 2006) was also computed, obtaining similar results.

By definition, the ICA may extract as many generators as the number of LFP signals. To correctly identify the presynaptic specificity of an IC, several conditions must be taken into account. First, each IC contributes differently to the total variance (power) of the LFP. Only those with a significant contribution ( $>1 \%$ in this work) were considered for further analysis. Second, the anatomic structure of each generator is fixed in each subject (Castellanos and Makarov, 2006; Korovaichuk et al., 2010; Makarov et al., 2010). In other words, the spatial profile of each ICs must be stable along the time. This was assessed by applying the ICA in different short-term epochs (Korovaichuk et al., 2010). Only those components present in all 
conditions with a stable spatial loading may represent true current generators. Moreover, a certain degree of similarity is expected between subjects and a specific pathway should have a comparable profile for different animals. Third, the synaptic specificity of each generator was determined by stimulating their respective excitatory pathways with subthreshold evoked activity (Makarova et al., 2011). Fourth, not every synaptic input leaves a footprint in the LFP. The geometry of the region and the distribution of axons and dendrites determines the real contribution of each pathway to the field potential (Buzsáki et al., 2012; Herreras, 2016; Herreras et al., 2015). This requires specific realistic models to test the multiple origins of the measured currents.

Applying ICA in our data recordings and considering all the conditions mentioned above, we were able to extract three common and stable generators in all subjects (Figure 1). They correspond to pathway-specific inputs to the hippocampus. Two of them were in CA1, one in str. radiatum, which correspond to the synaptic terminals of Schaffer collaterals from CA3 to the pyramidal cells in CA1 (Benito et al., 2014; Fernández-Ruiz et al., 2012a; Korovaichuk et al., 2010; Makarova et al., 2011; Martín-Vázquez et al., 2016; Schomburg et al., 2014) (SchIC). The other component had a current sink in str. lacunosum-moleculare ( $1 \mathrm{~m}$-IC), where are located the inputs from entorhinal cortex layer III (EC3) to the pyramidal cells in CA1 (Benito et al., 2014; Martín-Vázquez et al., 2016; Schomburg et al., 2014). A third component was identified in the DG, which corresponded to the axons projected from the EC layer II (EC2) to the dendrites of the granular cells through the perforant-pathway (Benito et al., 2014; Korovaichuk et al., 2010; Makarova et al., 2011) (PP-IC). Note that the active synaptic domain of PP-IC was in the molecular layer of the DG, but its field potential was dominant in the hilar region (Figure 1). This was generated by the volume conduction of the cell membranes in the molecular layer. The field potential of common currents, above and below the hilus, are overlapped in this region, thus increasing their electric field (Benito et al., 2014; Herreras, 2016; Herreras et al., 2015).

The extracted ICs represent the current sources of specific pathways to the hippocampus. Therefore, the temporal dynamics and rhythms of each generator reflects the activity generated in different nearby regions (CA3, EC3 and EC2 for Sch-IC, lm-IC and PP-IC, respectively). One limitation of this approach is that it cannot separate distinct temporal patterns within the same origin (i.e. theta and gamma oscillations). This is the consequence of two main effects. First, the same neuron could fire in multiple modes (Vinogradova, 2001). Moreover, the 
currents generated by synaptic terminals from the same region are fully overlapped in the space and their combination made up a single generator.

Another consideration is that the strongest generators could introduce contamination in other components (Korovaichuk et al., 2010; Schomburg et al., 2014). To ensure that the huge theta power is not affecting the discrimination of ICs, we separate the LFP in slow and fast rhythms by filtering the raw LFPs at $30 \mathrm{~Hz}$ (low-pass at $<30 \mathrm{~Hz}$ and a high-pass at $>30 \mathrm{~Hz}$, respectively). The ICA was applied to each filtered data-set separately. We compared the resultant ICs with those using the unfiltered data, confirming that the same generators were found in all conditions with quite similar time-series (Figure S2).

ICA does not ensure the correct polarity and amplitude of each generator. However, as the ICA algorithm is invertible, the LFPs generated by each component can be retrieved separately. The CSD can be applied to these reconstructed signals, obtaining the sinks and sources of each specific pathway. Such partial signals do have the correct polarity and amplitude (Korovaichuk et al., 2010; Martín-Vázquez et al., 2013).

\section{Preprocessing and power analysis of time series}

After computing the ICA algorithm, the dataset corresponding to each subject was composed by three time-series. These signals were downsampled at $625 \mathrm{~Hz}$ to improve the speed of computational analysis. They were also normalized, imposing to each dataset an averaged mean value of zero and a standard deviation of one to each signal separately. This way, we increase the similarities inter-subject and facilitate their comparison.

Power spectra were estimated using the multitaper method (Thomson, 1982). For power analysis at specific frequency bands we used an approach based on filtering and Hilbert transform (Jackson et al., 2006; Ólafsdóttir et al., 2017). First, the signal is bandpass filtered with a FIR filter between the frequencies of interest. Then, we computed the Hilbert transform and the instantaneous power was estimated as the squared complex modulus of the signal at each time point. The mean value was obtained as the averaged power in a certain time window. We defined the following frequency bands which are used along the text (unless otherwise indicated): delta (1-4 Hz), theta $(6-10 \mathrm{~Hz})$, slow gamma $(30-60 \mathrm{~Hz})$, medium gamma $(60-100$ $\mathrm{Hz})$ and fast gamma $(100-150 \mathrm{~Hz})$.

The linear interaction between IC-LFPs at each specific frequency was assessed using a coherence analysis. It measures the ratio between the cross power spectral density and their individual power spectral densities and was computed using the mscohere.m function in 
Matlab. The statistical significance was determined by a surrogate analysis (1000 surrogates in this work). With this methodology, the temporal relationship between signals was broken by randomly displacing one signal respect to the other. Then, the coherence surrogated results at each frequency were approximated to a Gaussian distribution and the significance threshold was the value for which the previous cumulative distribution was $0.95(\mathrm{p}=0.05)$.

To evaluate the distribution of the gamma activity along the phase of theta, the signals were first filtered at the frequencies of interest (gamma and theta) and the amplitude and phase were extracted using the Hilbert transform. Then, for each theta cycle, the envelope of the gamma activity was divided into $\mathrm{N}$ equidistant bins; an average along all cycles was then taken. Similar to the coherence analysis, the statistical significance was assessed by a surrogate analysis (1000 surrogates), randomly shifting the gamma signal with respect to the theta phase. The surrogated distribution was estimated by averaging the results of all simulations.

\section{Detection of theta rhythm}

From the whole recordings, only those epochs with a real theta rhythm in all components where considered for further analysis, that is, with high power at that band. Moreover, as oscillations with low amplitude could result in a less accurate estimation of their phase, we selected only those cycles with a minimum value of theta power to avoid this issue. To find such threshold we have modeled theta rhythm data as the combination of theta oscillations $\left(X_{\theta}(t)\right)$ and pink noise $\left(X^{n}(t)\right)$ to simulate the neural noise of the recordings:

$$
X_{\theta}^{n}(t)=X^{n}(t)+X_{\theta}(t)
$$

The noise was computed using the function pinknoise from Matlab, while $X_{\theta}(t)$ was composed by $d$ segments or cycles defined as:

$$
S_{i}^{k}\left(t_{i}\right)=A\left(\sin \left(2 \pi f_{i} t_{i}+1.5 \pi\right)\right)
$$

Where $k=1,2, \ldots, d$ and $f_{i} t_{i}$ where selected in order to that cycle had a duration of $T_{i} \in$ $[0.1,0.145]$ seconds, randomly chosen from a normal distribution with mean 0.125 and standard deviation 0.02 .

Knowing each $S_{i}^{k}\left(t_{i}\right)$, we could estimate perfectly the phase of the theta rhythm, i.e. this was the ground truth. Briefly, for each simulation we changed the relative power between both components by varying the $A$ parameter and we estimated the phase of the theta oscillation (see below). Doing so, we were able to measure the error between our estimation and the 
ground truth as a function of the relative theta power and then find the value that minimizes that error. A detailed description of these steps follows.

For each dataset, we bandpass filtered each signal at delta $(1-4 \mathrm{~Hz})$ and theta $(6-10 \mathrm{~Hz})$ frequencies. Then, we computed the Hilbert transform and the instantaneous power was estimated as the squared complex modulus of the signal at each time point. The relative theta power was obtained as the ratio between the averaged theta by the delta power (Jackson et al., 2006; Ólafsdóttir et al., 2017). The phase of the oscillation was estimated for the theta filtered signal through Hilbert, being zero and $\pi$ radians the values corresponding to the trough and the peak of the cycle, respectively. Finally, the error was measured as the averaged distance (in milliseconds) between each trough of the real phase and the estimated one. Additionally, we computed the minimum error as that obtained when $X^{n}(t)$ is set to zero. This value corresponds to the noise introduced by the method used to estimate the phase and does not depend on the power. The relationship between the ratio and the error is shown in Figure S3.

Using the simulated data, we considered that the theta power was not influencing the estimation of the theta phase when the error due to the amplitude (i.e. not considering the one introduced by the filtering) was lower than $1 \mathrm{~ms}$. This corresponded to a ratio of theta power 3.78 times (we took 4 for simplicity) higher than delta (Figure S3). In the real recordings we expect not only neural noise but also activity at the delta band. Thus, the theoretical threshold obtained by this procedure represent a conservative measurement. For those cases with high delta activity, the threshold would be more restrictive as the ratio decreases, but the theta power would be always high enough to guarantee a correct estimation of the phase.

To detect the theta rhythm in all the ICs recorded, we used a sliding window of one theta cycle (approximately $125 \mathrm{~ms}$ ) and selected only those epochs where the ratio between their theta power by delta (computed as described above) was higher than 4. Moreover, analysis taken thresholds of 6 and 8 were also done for comparison purposes, showing no significant differences.

\section{Optogenetic modulation of pathway-specific theta activity.}

In the Cre-expressing transgenic subjects we analyzed whether the light stimulation of CA3 PV-interneurons had an effect exclusively in the Schaffer collateral theta output. We compared time windows of two seconds immediately after the stimulation with the first two seconds of the stimulus. This period was chosen to minimize the influence of different locomotor activities between windows. For all trials, only those with theta oscillation (see above) were further 
considered. Then, we computed the power spectrum of each IC-LFP using a multitaper approach (Thomson, 1982).

\section{Inter-cycle phase clustering}

To estimate the relative theta phase between ICs, we used a modified inter-trial phase clustering approach (Cohen, 2014) to account for differences between cycles instead of trials (inter-cycle phase clustering, ICPC). In this methodology, each trial is defined as a vector with modulus one and the angle corresponding to the phase of the oscillation measured at a specific time point $\left(\varphi_{t}\right)$. Then, the ICPC is computed as the modulus of the averaged vector among all trials:

$$
I C P C=\left|\frac{1}{N} \sum_{t=1}^{N} e^{i \varphi_{t}}\right|
$$

If the distribution of angles is uniform along the polar axis, then the ICPC value is zero. On the contrary, values near one indicate a preferred phase in the distribution, being one when all trials have the same phase.

For the analysis in Figure 1h, the phases of each signal were extracted following (Cole and Voytek, 2019) to have a better characterization of the shape of the theta rhythm. The LFP measured in the pyramidal layer of CA1 (pyr. CA1) was considered as reference, where its trough and peak coincide with 0 and $\pi$ radians, respectively. We calculated the ICPC for the different components separately, where each trial was the phase of the IC measured at each trough of pyr. CA1. Thus, the number of trials corresponded to the number of theta cycles in pyr. CA1 and the angle and value of the ICPC can be interpreted as the phase difference and the stability of the IC with respect to pyr. CA1, respectively.

The statistical significance was assessed by a surrogate analysis (1000 surrogates in this work), randomly shifting the phase of the IC and keeping pyr. CA1 constant. For each simulated dataset, the ICPC was computed fitting all the surrogated results into a normal distribution. Then, the p-value associated to the ICPC of the IC was obtained as 1 minus the previous normal cumulative distribution evaluated at the ICPC value.

\section{Cycle-by-cycle synchronization using ICPC}

Using the ICPC approach, the degree of coherence between theta rhythms was estimated for each cycle separately. Doing this process, a dynamic measurement of synchronization can be done identifying time epochs of high and low coherence. Considering two ICs, one acting as 
the reference, the ICPC value of each theta cycle was computed using only three cycles, which corresponded to the relative phase at that cycle and the previous and consecutive ones (Figure S4). If the waves were highly coherent, then their phase would be similar along theta cycles, resulting in an ICPC value close to 1; for those with different phases, the ICPC would be lower. In this work, we considered the theta oscillation in $\mathrm{lm}$-IC as the reference to compute the ICPC, as it had the highest amplitude at that frequency. Moreover, the ICPC between pairs of signals (i.e. $\mathrm{lm}$-IC vs. Sch-IC and $\mathrm{lm}$-IC vs. PP-IC) was averaged as an approximation of the global synchronization of the network at each time epoch.

The instantaneous ICPC can be compared to other metrics as frequency or power, analyzing the correlation between synchronized state and the features of the signals. To compute each correlation, the data was classified into 10 groups as a function of the ICPC, with ten equidistant bins from 0.75 to 1 . We chose these values as they contain at least the $90 \%$ of the cycles in all subjects and 80 cycles per bin (around 10 seconds). Then, the averaged value of the different metrics was computed for each group, analyzing the correlation between the means and the ICPC value. To identify reliably relationships, we compared if the resultant correlation value $(\rho)$ was higher than the obtained by a surrogate analysis. Each simulated dataset (100 surrogates in this work) was built by randomly shifting the IC components, breaking any temporal relationship between them. Then, the correlation between the ICPC and other features of interest were computed, fitting the results to a gaussian distribution. We considered that a correlation was significant if its value was higher than the $95^{\text {th }}$ percentile of the surrogated distribution.

\section{$\underline{\text { Multiple linear regression }}$}

The joint analysis of several features as predictors of the ICPC was done using a multiple linear regression (MLR). Firstly, we ranked all the values associated to each feature (function tiedrank.m in matlab) to minimize the effect of outlayers in the dataset. Then, a single MLT is fitted using each predictor multiplied by a beta factor:

$$
I C P C=\beta_{0}+\beta_{1} \text { power }+\beta_{2} \text { frequency }+\beta_{3} \text { speed }+\cdots+\varepsilon
$$

Where $\beta_{0}$ is a constant value and $\varepsilon$ are the residuals.

The contribution of one specific predictor to the total explained variance of the model is estimated by fitting a reduced MLR without that predictor and computing the difference between variances in the full model minus the reduced one. 
To estimate if each variable is significantly contributing to the ICPC across subjects, we followed (Montgomery and Buzsáki, 2007) and we tested their associated beta values for a statistical difference from zero (t-test, Bonferroni corrected).

\section{Cross-Frequency Coupling}

Interactions between the phase of a low frequency oscillation and the amplitude of a faster one were measured using an approach based on the modulation index (MI) (Canolty et al., 2006; Tort et al., 2008). The original method computed the phases and amplitudes through filtering and Hilbert transform. Nevertheless, theta rhythms in the hippocampus are non-sinusoidal oscillations and filtering the signal results in errors at estimating the waveform shape and could introduce spurious coupling (Cole and Voytek, 2017; Kramer et al., 2008). A new methodology has been proposed to overcome this issue and estimate the instantaneous phase of this kind of oscillations (Cole and Voytek, 2019). Briefly, we used a combination of a narrowband filter to detect the zero-crossing points (which correspond to the ascendant and descendant slope of the oscillation, or the phases $\pi / 2$ and $3 \pi / 3$, respectively) and a broadband filter to find the trough and the peak (phases 0 and $\pi$, respectively). This way, the phase of the signal does not vary monotonically (as in the case of a sinusoidal one) but could follow fast changes in the cycle as an abrupt ascendant slope and a soft descent. We named this signal as $x_{\theta \varphi}(t)$.

The amplitude of the faster oscillation is computed as the envelope of the signal filtered at the specific frequency which we want to analyze. First, we used a filter centered at that frequency and with a bandwidth at least two times the frequency of the phase signal where the coupling is expected to be maximum (Aru et al., 2015). Considering that the main rhythm in the hippocampus is around $8 \mathrm{~Hz}$, the bandwidth should have at least $16 \mathrm{~Hz}$ (we used $20 \mathrm{~Hz}$ in this work). Then, the envelope is computed using the Hilbert transform. The resultant signal is labelled as $x_{\gamma A}(t)$.

To compute the MI, we divide each cycle of $x_{\theta \varphi}(t)$ into $N$ bins. To avoid the issues introduced in this method due to the previously mentioned non-uniform theta oscillation (Cole and Voytek, 2017; van Driel et al., 2015), these bins were not of the same size, but were equalized along the phase of the theta cycle. Instead of using $N$ bins, we divided each cycle into 4 segments which correspond to the epochs between the peak, the trough and both ascendant and descendant pendants (Cole and Voytek, 2019), and then divided that segment into N/4 bins of the same size. Therefore, N/4 bins were used from the trough to the middle of the ascendant phase, N/4 bins from this point to the peak and so on. After that, we computed the mean 
amplitude of $x_{\gamma A}(t)$ at each bin, calling $\left\langle x_{\gamma A}\right\rangle_{\varphi}(j)$ the amplitude at the phase bin $j$. From them, we can calculate the entropy $H$, defined by:

$$
H=-\sum_{j=1}^{N} p_{j} \log p_{j}
$$

where $N$ was set to 20 , and $p_{j}$ is given by

$$
p_{j}=\frac{<x_{\gamma A}>_{\varphi}(j)}{\sum_{j=1}^{N}<x_{\gamma A}>_{\varphi}(j)}
$$

The value of MI is obtained normalizing $H$ by the maximum entropy $\left(H_{\max }\right)$, given by the uniform distribution $p_{j}=1 / N$ (i.e. $H_{\max }=\log N$ ):

$$
M I=\frac{H_{\max }-H}{H_{\max }}
$$

A value of MI near to 0 indicates lack of phase-to-amplitude modulation, while larger MI values reflect higher coupling between both signals. The statistical significance has been assessed following the steps proposed by Canolty and colleagues (Canolty et al., 2006), by a surrogate analysis ( $\mathrm{n}=100$ surrogates) in which each surrogate is built by cutting the phase signal at a random point and exchanging the resultant segments. This breaks the temporal relationship between both time-series minimizing the distortion of their dynamics (Aru et al., 2015). The MI estimated among surrogates represents the coupling due to the oscillatory nature of the signals but not by a real temporal relationship. Therefore, the MI values of all surrogates are approximated to a gaussian distribution, whose 95th percentile is considered as a significance threshold.

\section{Cross-Frequency Directionality}

The MI is a measurement of the degree of interaction between the phase and the amplitude of two frequencies, but it has no information regarding the directionality in this coupling. On the one hand, the theta phase would modulate the amplitude at gamma frequencies while, on the other hand, the gamma activity could be leading the phase. To identify leader and follower in this interaction, we have used the cross-frequency directionality (CFD) index (Jiang et al., 2015). The main idea is that, supposing that the phase component precedes the amplitude with a fixed time delay of $k \mathrm{~ms}$, the distance from the peak of the phase to the next peak of the amplitude should be $k$ ms (i.e. there is an increase of gamma activity $k \mathrm{~ms}$ after the peak of theta phase). Thus, as not all phase cycles have the same duration, the distance from the peak 
of the gamma amplitude to the next peak of theta may vary. Nevertheless, in the case of gamma activity preceding theta, the result would be the opposite. The peak of gamma activity should appear $k \mathrm{~ms}$ before the peak of the phase, while the timing from phase to amplitude would be different for each cycle. Note that the CFD is an estimation of the temporal relations between signals, but not a measurement of causality per se as, for example, a third source could be interacting with both signals.

The CFD identifies this relationship using the phase-slope index (Nolte et al., 2008) (PSI), a measurement of directionality between time series. Briefly, if the oscillation of one signal $x(t)$ at a certain frequency is driving a second one $y(t)$ with a time delay, then the phase difference between them at that specific delay will change consistently with the frequency of the signals. The slope of the phase is obtained in function of the frequency, and its sign will indicate who is the driver. If the slope is positive (higher the frequency, higher the phase difference between signals) then $x(t)$ leads $y(t)$ in time, while negative values would indicate that $y(t)$ precedes $x(t)$. The CFD is a variant of the PSI, where one signal is the theta component, and the other the envelope of the gamma activity.

Calling $x(t)$ to the original signal, $x_{\gamma A}^{v}(t)$ to the power envelope of the signal at a $v$ gamma frequency, and being $X$ and $X_{\gamma A}^{v}$ their Fourier transform, respectively, the CFD is defined as the PSI by:

$$
\psi\left(v, f_{j}\right)=\operatorname{Im}\left(\sum_{f_{j}-\frac{\beta}{2}}^{f_{j}+\frac{\beta}{2}} C^{*}\left(v, f_{j}\right) C\left(v,\left(f_{j}+\Delta f\right)\right)\right)
$$

where

$$
C\left(v, f_{j}\right)=\frac{\sum_{S=1}^{S} X^{S}\left(X_{\gamma A}^{v, S}\right)^{*}}{\sqrt{\sum_{S=1}^{S}\left|X^{S}\right|^{2} \sum_{S=1}^{S}\left|X_{\gamma A}^{v, S}\right|^{2}}}
$$

is the complex coherency, $f_{j}$ is the theta frequency under study, $S$ is the number of segments in which the signal has been divided and $\beta$ is the bandwidth for which the phase slope is measured, and it has been fixed at $2 \mathrm{~Hz}, 4$ times the resolution $(\Delta f=0.5 \mathrm{~Hz})$.

This methodology has been proposed specifically for those frameworks with phase-amplitude coupling as more classical approaches like Granger Causality (Granger, 1969) have some unavoidable limitations due to the use of filters (Barnett and Seth, 2011) and the differences of 
signal-to-noise ratio in both components (Nolte et al., 2010), which provoke that they cannot identify the correct directionality in CFC models (Jiang et al., 2015).

To provide statistical significance, a new surrogate test ( $\mathrm{n}=100$ surrogates) has been developed following the same steps than in the MI analysis. Note that in this case, two thresholds are imposed, considering both tails of the gaussian distribution (i.e. positive and negative values). In this work, we have used the Matlab toolbox HERMES (Niso et al., 2013) and the implementation of PSI in Matlab code (http://doc.ml.tu-berlin.de/causality/). To emphasize the directionality in the region of higher CFC, the MI comodulogram has been redefined as a mask, with values from 0 to 1 (minimum and maximum value MI). Applying this mask to the CFD comodulogram, areas without phase-amplitude coupling will be attenuated, while the main cluster remains constant. 


\section{Supplementary Bibliography}

Andersen P, Holmqvist B, Voorhoeve PE. 1966. Entorhinal activation of dentate granule cells. Acta Physiol Scand 66:448-460. doi:10.1111/j.1748-1716.1966.tb03223.x

Aru Juhan, Aru Jaan, Priesemann V, Wibral M, Lana L, Pipa G, Singer W, Vicente R. 2015. Untangling cross-frequency coupling in neuroscience. Curr Opin Neurobiol, SI: Brain rhythms and dynamic coordination 31:51-61. doi:10.1016/j.conb.2014.08.002

Barnett L, Seth AK. 2011. Behaviour of Granger causality under filtering: theoretical invariance and practical application. J Neurosci Methods 201:404-419. doi:10.1016/j.jneumeth.2011.08.010

Bell AJ, Sejnowski TJ. 1995. An information-maximization approach to blind separation and blind deconvolution. Neural Comput 7:1129-1159.

Benito N, Fernández-Ruiz A, Makarov VA, Makarova J, Korovaichuk A, Herreras O. 2014. Spatial modules of coherent activity in pathway-specific LFPs in the hippocampus reflect topology and different modes of presynaptic synchronization. Cereb Cortex N Y N 1991 24:1738-1752. doi:10.1093/cercor/bht022

Buzsáki G, Anastassiou CA, Koch C. 2012. The origin of extracellular fields and currents--EEG, ECoG, LFP and spikes. Nat Rev Neurosci 13:407-420. doi:10.1038/nrn3241

Canolty RT, Edwards E, Dalal SS, Soltani M, Nagarajan SS, Kirsch HE, Berger MS, Barbaro NM, Knight RT. 2006. High Gamma Power Is Phase-Locked to Theta Oscillations in Human Neocortex. Science 313:1626-1628. doi:10.1126/science.1128115

Castellanos NP, Makarov VA. 2006. Recovering EEG brain signals: Artifact suppression with wavelet enhanced independent component analysis. J Neurosci Methods 158:300-312. doi:10.1016/j.jneumeth.2006.05.033

Chen A. 2006. Fast Kernel Density Independent Component AnalysisIndependent Component Analysis and Blind Signal Separation, Lecture Notes in Computer Science. Springer, Berlin, Heidelberg. pp. 24-31. doi:10.1007/11679363_4

Cohen MX. 2014. Analyzing Neural Time Series Data. Cambridge: Mit Press.

Cole S, Voytek B. 2019. Cycle-by-cycle analysis of neural oscillations. I Neurophysiol. doi:10.1152/jn.00273.2019

Cole SR, Voytek B. 2017. Brain Oscillations and the Importance of Waveform Shape. Trends Cogn Sci 21:137-149. doi:10.1016/j.tics.2016.12.008

Fernández-Ruiz A, Makarov VA, Benito N, Herreras O. 2012a. Schaffer-specific local field potentials reflect discrete excitatory events at gamma frequency that may fire postsynaptic hippocampal CA1 units. J Neurosci Off J Soc Neurosci 32:5165-5176. doi:10.1523/JNEUROSCI.4499-11.2012

Fernández-Ruiz A, Makarov VA, Herreras O. 2012b. Sustained increase of spontaneous input and spike transfer in the CA3-CA1 pathway following long-term potentiation in vivo. Front Neural Circuits 6. doi:10.3389/fncir.2012.00071

Freeman JA, Nicholson C. 1975. Experimental optimization of current source-density technique for anuran cerebellum. J Neurophysio/ 38:369-382. doi:10.1152/jn.1975.38.2.369

Granger CWJ. 1969. Investigating Causal Relations by Econometric Models and Cross-spectral Methods. Econometrica 37:424-438. doi:10.2307/1912791

Herreras O. 2016. Local Field Potentials: Myths and Misunderstandings. Front Neural Circuits 10:101. doi:10.3389/fncir.2016.00101

Herreras O. 1990. Propagating dendritic action potential mediates synaptic transmission in CA1 pyramidal cells in situ. J Neurophysiol 64:1429-1441. doi:10.1152/jn.1990.64.5.1429

Herreras O, Makarova J, Makarov VA. 2015. New uses of LFPs: Pathway-specific threads obtained through spatial discrimination. Neuroscience 310:486-503. doi:10.1016/j.neuroscience.2015.09.054

Holsheimer J. 1987. Electrical conductivity of the hippocampal CA1 layers and application to currentsource-density analysis. Exp Brain Res 67:402-410. 
Jackson JC, Johnson A, Redish AD. 2006. Hippocampal sharp waves and reactivation during awake states depend on repeated sequential experience. J Neurosci Off J Soc Neurosci 26:1241512426. doi:10.1523/JNEUROSCI.4118-06.2006

Jiang H, Bahramisharif A, van Gerven MAJ, Jensen O. 2015. Measuring directionality between neuronal oscillations of different frequencies. Neurolmage 118:359-367. doi:10.1016/j.neuroimage.2015.05.044

Korovaichuk A, Makarova J, Makarov VA, Benito N, Herreras O. 2010. Minor contribution of principal excitatory pathways to hippocampal LFPs in the anesthetized rat: a combined independent component and current source density study. I Neurophysiol 104:484-497. doi:10.1152/jn.00297.2010

Kramer MA, Tort ABL, Kopell NJ. 2008. Sharp edge artifacts and spurious coupling in EEG frequency comodulation measures. J Neurosci Methods 170:352-357. doi:10.1016/j.jneumeth.2008.01.020

López-Aguado L, Ibarz JM, Herreras O. 2001. Activity-dependent changes of tissue resistivity in the CA1 region in vivo are layer-specific: modulation of evoked potentials. Neuroscience 108:249262.

Makarov VA, Makarova J, Herreras O. 2010. Disentanglement of local field potential sources by independent component analysis. J Comput Neurosci 29:445-457. doi:10.1007/s10827-0090206-y

Makarova J, Ibarz JM, Makarov VA, Benito N, Herreras O. 2011. Parallel readout of pathway-specific inputs to laminated brain structures. Front Syst Neurosci 5:77. doi:10.3389/fnsys.2011.00077

Martín-Vázquez G, Benito N, Makarov VA, Herreras O, Makarova J. 2016. Diversity of LFPs Activated in Different Target Regions by a Common CA3 Input. Cereb Cortex N Y N 1991 26:4082-4100. doi:10.1093/cercor/bhv211

Martín-Vázquez G, Makarova J, Makarov VA, Herreras O. 2013. Determining the true polarity and amplitude of synaptic currents underlying gamma oscillations of local field potentials. PloS One 8:e75499. doi:10.1371/journal.pone.0075499

Mitzdorf U. 1985. Current source-density method and application in cat cerebral cortex: investigation of evoked potentials and EEG phenomena. Physiol Rev 65:37-100. doi:10.1152/physrev.1985.65.1.37

Montgomery SM, Buzsáki G. 2007. Gamma oscillations dynamically couple hippocampal CA3 and CA1 regions during memory task performance. Proc Natl Acad Sci U S A 104:14495-14500. doi:10.1073/pnas.0701826104

Niso G, Bruña R, Pereda E, Gutiérrez R, Bajo R, Maestú F, del-Pozo F. 2013. HERMES: towards an integrated toolbox to characterize functional and effective brain connectivity. Neuroinformatics 11:405-434. doi:10.1007/s12021-013-9186-1

Nolte G, Ziehe A, Krämer N, Popescu F, Müller K-R. 2010. Comparison of Granger Causality and Phase Slope IndexCausality: Objectives and Assessment. Presented at the Causality: Objectives and Assessment. pp. 267-276.

Nolte G, Ziehe A, Nikulin VV, Schlögl A, Krämer N, Brismar T, Müller K-R. 2008. Robustly estimating the flow direction of information in complex physical systems. Phys Rev Lett 100:234101. doi:10.1103/PhysRevLett.100.234101

Ólafsdóttir HF, Carpenter F, Barry C. 2017. Task Demands Predict a Dynamic Switch in the Content of Awake Hippocampal Replay. Neuron 96:925-935.e6. doi:10.1016/j.neuron.2017.09.035

Schomburg EW, Fernández-Ruiz A, Mizuseki K, Berényi A, Anastassiou CA, Koch C, Buzsáki G. 2014. Theta Phase Segregation of Input-Specific Gamma Patterns in Entorhinal-Hippocampal Networks. Neuron 84:470-485. doi:10.1016/j.neuron.2014.08.051

Thomson DJ. 1982. Spectrum estimation and harmonic analysis. Proc IEEE 70:1055-1096. doi:10.1109/PROC.1982.12433

Tort ABL, Kramer MA, Thorn C, Gibson DJ, Kubota Y, Graybiel AM, Kopell NJ. 2008. Dynamic crossfrequency couplings of local field potential oscillations in rat striatum and hippocampus during 
performance of a T-maze task. Proc Natl Acad Sci U S A 105:20517-20522. doi:10.1073/pnas.0810524105

van Driel J, Cox R, Cohen MX. 2015. Phase-clustering bias in phase-amplitude cross-frequency coupling and its removal. J Neurosci Methods 254:60-72. doi:10.1016/j.jneumeth.2015.07.014

Vinogradova OS. 2001. Hippocampus as comparator: role of the two input and two output systems of the hippocampus in selection and registration of information. Hippocampus 11:578-598. doi:10.1002/hipo.1073

Wood ER, Dudchenko PA, Robitsek RJ, Eichenbaum H. 2000. Hippocampal neurons encode information about different types of memory episodes occurring in the same location. Neuron 27:623-633. 


\section{A}
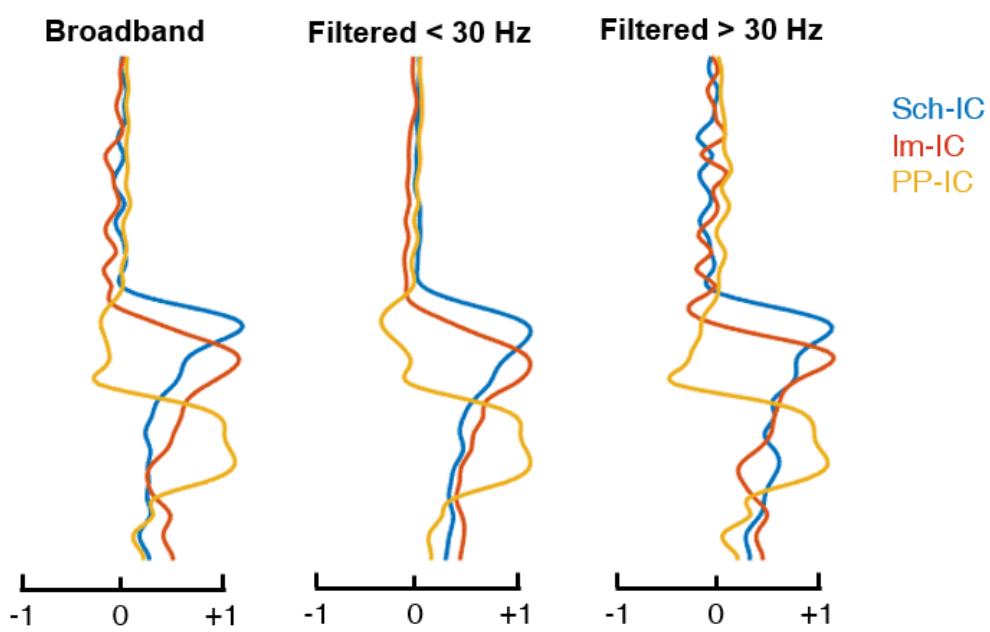

B

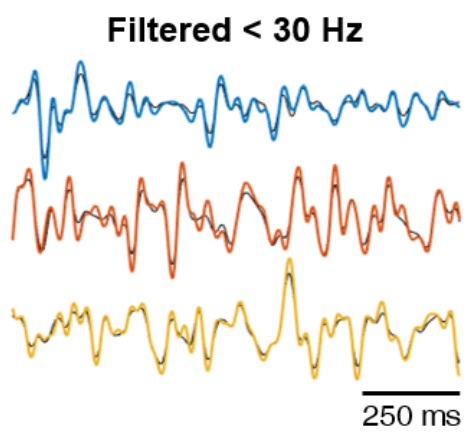

Filtered $>30 \mathrm{~Hz}$

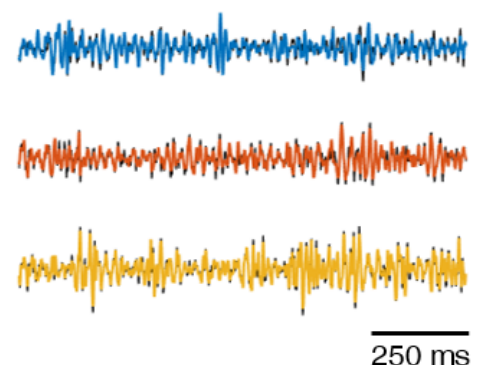

Sch-IC filtered

Sch-IC broadband

Im-IC filtered

Im-IC broadband

PP-IC filtered

PP-IC broadband

Figure S1: Signal pre-processing minimally affects the ICA results. (A) Voltage loadings obtained from an ICA applied to the raw (broadband) LFP recording (left), to the same LFP but low-pass filtered at 30 $\mathrm{Hz}$ (centre) or high-pass filtered at $30 \mathrm{~Hz}$ (right). In all cases, the three main components share a common spatial pattern, with maximums at their afferent hippocampal pathway. (B) Examples of time series comparing the IC-LFP extracted by computing the ICA algorithm and then filtering the signal (black traces), versus those obtained by filtering first the LFPs and then applying ICA (colour-coded traces). There are no significant differences neither in the phase of the theta rhythm (center, low-pass filtered at $30 \mathrm{~Hz}$ ) nor in the amplitude of gamma oscillations (right, high-pass filtered at $30 \mathrm{~Hz}$ ). 
a

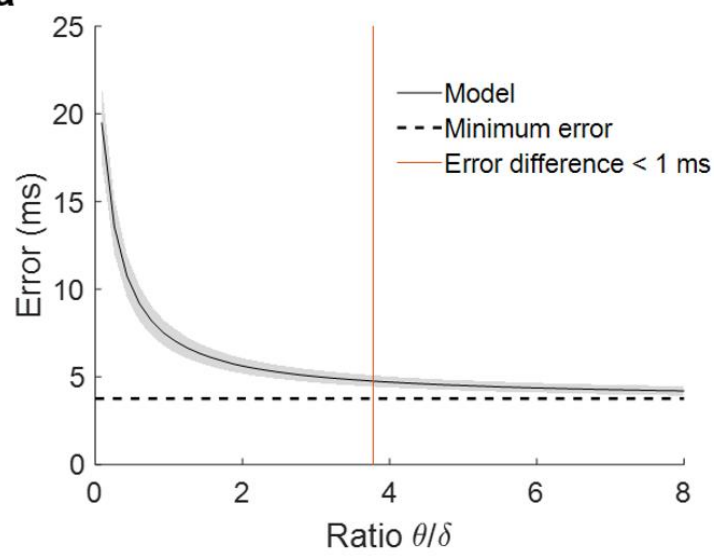

b

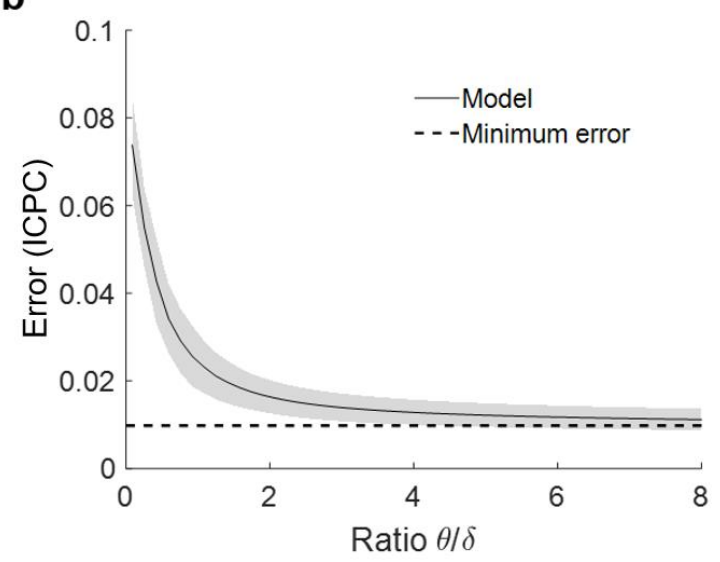

Figure S2: (a) Phase estimation error for simulations with different ratio between theta and delta power. Each point of the solid black line represents the mean error and the grey shadow the standard deviation over 100 simulations. The dashed line is the minimum error, when no noise is added to the model and the estimation does not depend on theta power. The red line marks the point where error due to power is lower than 1 millisecond (ratio = 3.78). (b) Estimation of the error in the ICPC between two signals as a function of the ratio between theta and delta, following the same methodology as in panel (a) to simulate the signals. For a ratio of 3.78 , the accuracy of the ICPC value has and error lower than \pm 0.01 . 

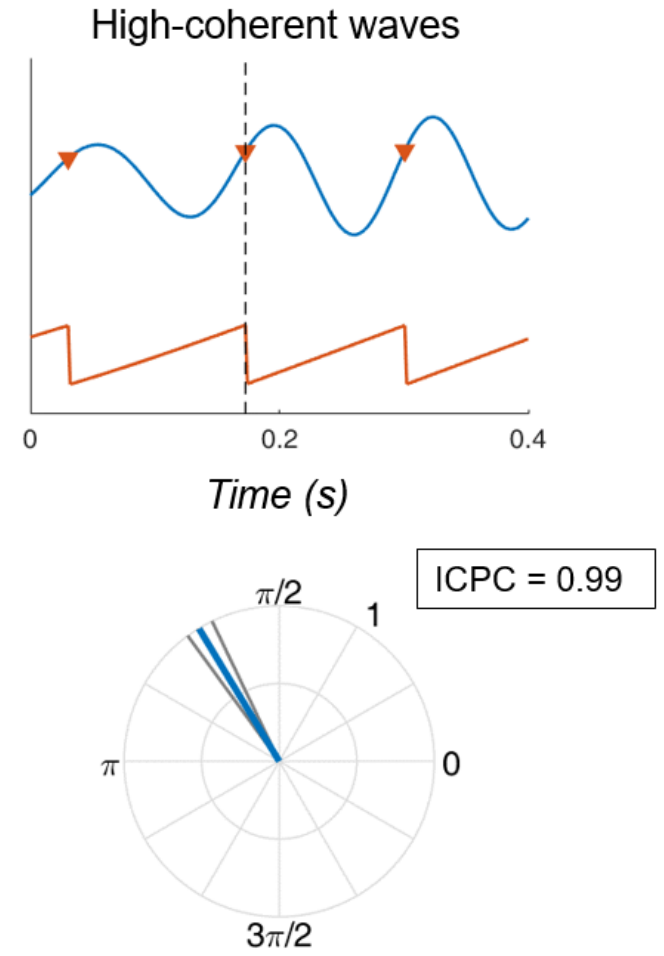

Low-coherent waves
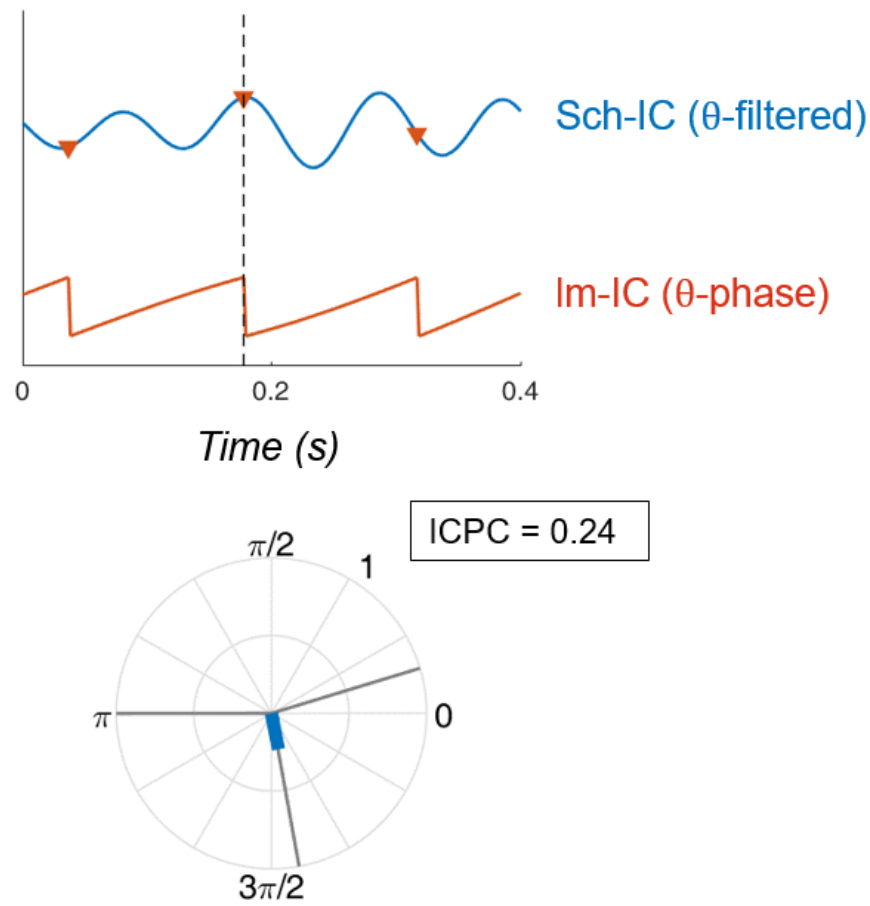

Figure S3: Examples of synchronization using ICPC. (Top) Temporal traces of Sch-IC (filtered at theta frequency) and the theta phase of $\mathrm{Im}-\mathrm{IC}$. Each step in the saw-tooth signal ( $\pi$ radians) is marked with a red triangle and corresponds to a peak in the theta oscillation of $\mathrm{Im}-\mathrm{IC}$. Black dashed lines indicate the cycle under consideration. (Bottom) The synchronization between signals is measured by computing the ICPC using the previous, present and consecutive cycles. 


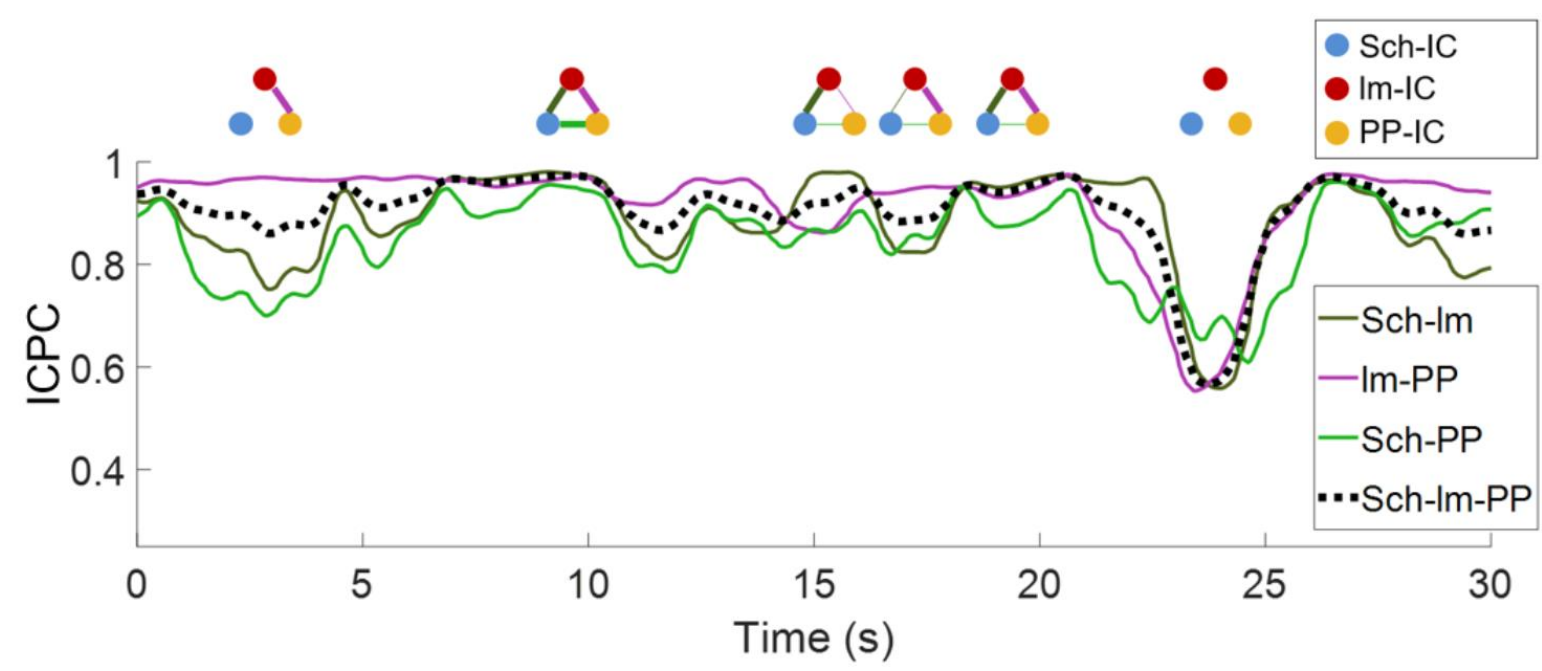

Figure S4: Representative values of ICPC in one animal along time. The dashed line represents the ICPC computed between the three IC-LFPs, while color-coded curves are the ICPC between pairs of IC-LFPs. A schematic diagram of the connectivity at specific time points is shown at the top, where the thickness of the lines represents the ICPC value between pairs. For clarity, epochs of lower ICPC are represented as nodes without links. 


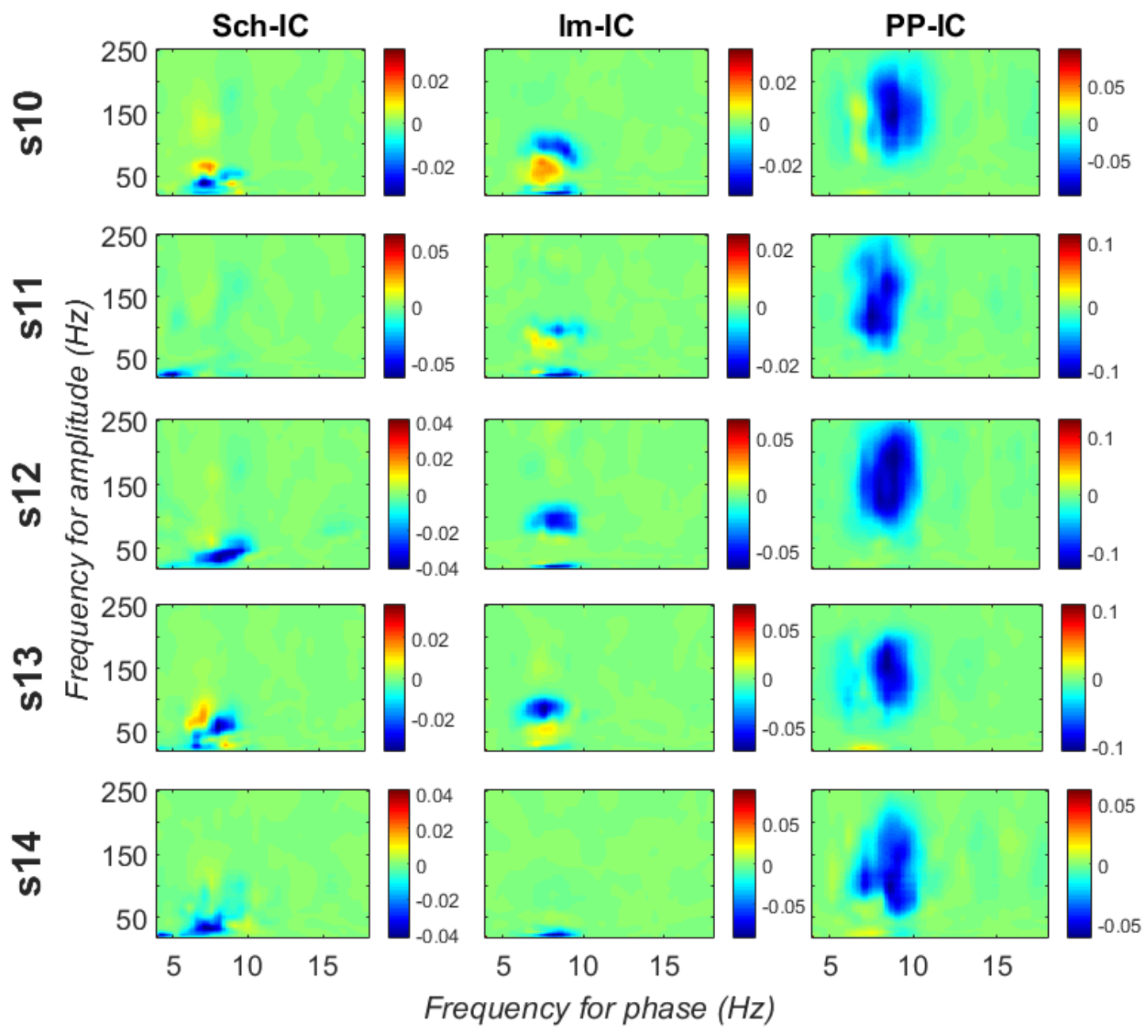

Figure S5: CFD for individual animals measured along all the recording time. Significant negative values (gamma amplitude drives theta phase) were found in 4 out of 5 subjects in Sch-IC and Im-IC for those pairs of frequencies with maximum CFC $(37.5 \pm 5 \mathrm{~Hz}$ in Sch-IC and $82.5 \pm 4 \mathrm{~Hz}$ in Im-IC), while negative CFDs were significant in PP-IC for all subjects. 

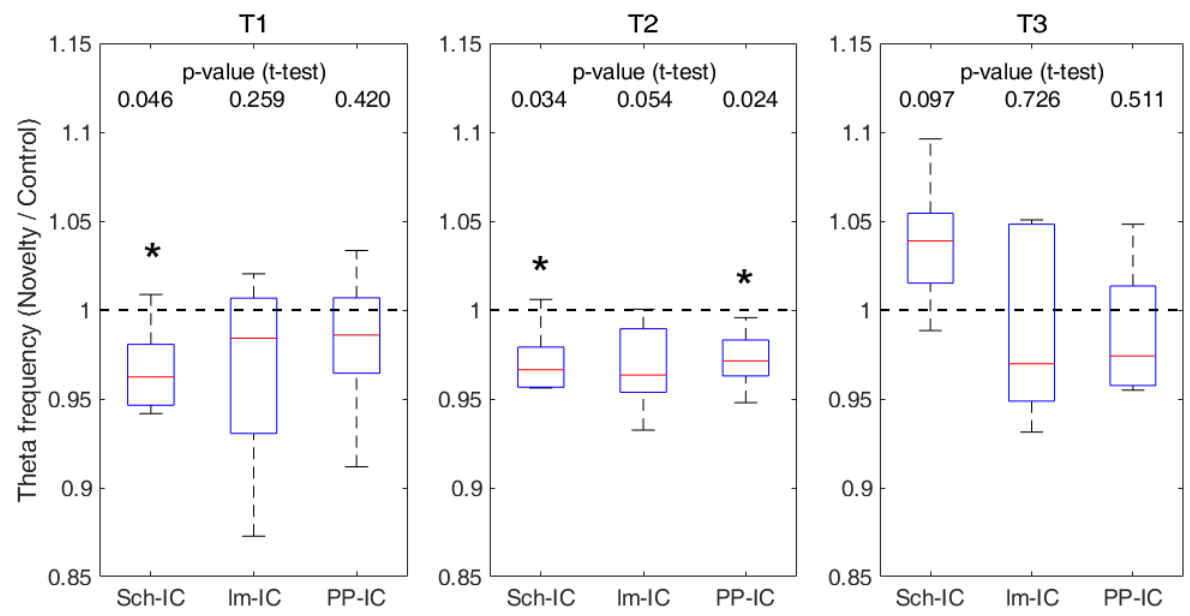

Figure S6: Ratio of theta frequency in novelty sessions with respect to control sessions and for three different time windows during the task $(* \mathrm{p}<0.05$, paired $\mathrm{t}$-test, $\mathrm{N}=5)$. 

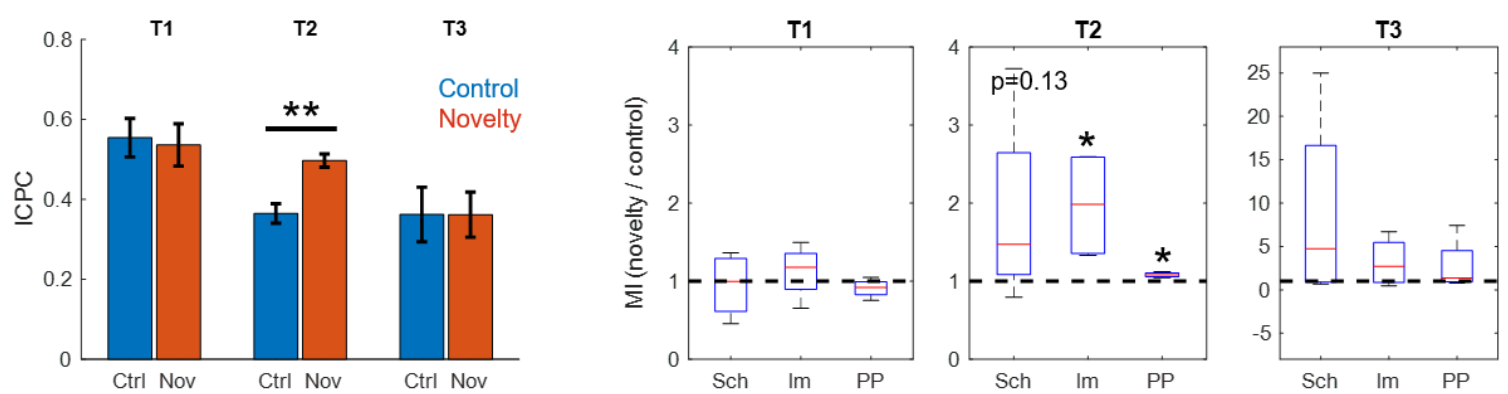

Figure S7: Comparison between ICPC (left, $* * p<0.01$, paired t-test between subjects, $t=7.86, \mathrm{~N}=4$ ) and $\mathrm{MI}$ (right) in control versus novelty sessions after discarding epochs of rearing in the recordings (* $\mathrm{p}<0.05$, paired $\mathrm{t}$-test between subjects, $\mathrm{t}=3.34 / 4.91$ for $\mathrm{Im}-\mathrm{IC} / \mathrm{PP}-\mathrm{IC}$ in $\mathrm{t} 2, \mathrm{~N}=4)$. 